\title{
The impact of a low-calorie, low-glycemic diet on systemic lupus erythematosus: a systematic review
}

\author{
Aline Mizusaki Imoto $\left.{ }^{1 *} \mathbb{(}\right)$, Leila Bernarda Gottems ${ }^{1} \mathbb{D}$, Ana Lúcia Salomon² ${ }^{\mathbb{D}}$, Helbert Eustáquio Cardoso e Silva ${ }^{1} \mathbb{D}$, \\ Império Lombardi Júnior ${ }^{3}$ (D) Maria Stella Peccin ${ }^{3}$ [D, Fábio Ferreira Amorim ${ }^{4,5}$ (B] and Levy Aniceto Santana ${ }^{1}$ (D)
}

\begin{abstract}
Background: Diet plays a critical role in Systemic Lupus Erythematosus (SLE) patients, impacting on the microbiota composition and, consequently, on the immune response. The objective was to analyze and verify the diet effect on SLE patients.

Methods: This is a systematic review performed at the Evidences-based Health Lab, Escola Superior em Ciências da Saúde, Brasília (DF), Brazil. In March, 2021, five databases, and grey literature, through JSTOR, Open Grey, and Google Scholar were searched. Randomized Clinical Trials in which SLE patients with calorie restricted, low glycemic index or other diet involving the joint adequacy of these aspects, compared with placebo or different types of diet, were included.

Results: It was identified in the databases 758 articles; 132 were duplicated; 616 references were screened, and 604 were excluded. After reading the title and abstract, 12 articles were included for full-text reading. After the full-text reading, three studies were included for quantitative analysis. The diet improved the quality of life at 6 (MD 16.30; $5.91 ; 26.69)$ and 12 weeks (MD 14.60; 0.88;28.32). The GRADE was used to evaluate the quality of evidence.
\end{abstract}

Conclusion: There is low evidence that the diet has a positive impact on the quality of life of SLE patients. Trial registration PROSPERO-CRD4202012208.

Keywords: Evidence based nutrition, Nutrition intervention, Lupus erythematosus management

\section{Introduction}

Systemic lupus erythematosus (SLE) can be defined as a chronic autoimmune disease with varied, complex clinical manifestations [1]. According to the Lupus Commission of Brazilian Society of Rheumatology, it is an estimated prevalence of 65,000 people diagnosed with systemic lupus erythematosus in Brazil [2]. The incidence

\footnotetext{
*Correspondence: alinem.imoto@gmail.com

${ }^{1}$ Evidences-Based Health Lab, Professional and Academic Master Program, Escola Superior em Ciências da Saúde, Brasília, DF, Brazil Full list of author information is available at the end of the article
}

is higher in women, 1 in 1,700 . In a systematic review (SR) of epidemiological studies by Rees et al. [3], the highest world incidence was verified in North America and the lowest in Africa. Furthermore, in most studies, European countries showed the lowest incidence, while Asia, Australia, and America showed the highest rates. It should be stressed that incidences may be associated with places where studies are more frequently conducted or more availability of treatment is confirmed.

Immune system activation in SLE is characterized by exaggerated $\mathrm{B}$-cell and $\mathrm{T}$-cell responses and loss of immune tolerance against self-antigens, contributing to original author(s) and the source, provide a link to the Creative Commons licence, and indicate if changes were made. The images or other third party material in this article are included in the article's Creative Commons licence, unless indicated otherwise in a credit line to the material. If material is not included in the article's Creative Commons licence and your intended use is not permitted by statutory regulation or exceeds the permitted use, you will need to obtain permission directly from the copyright holder. To view a copy of this licence, visit http://creativecommons.org/licenses/by/4.0/. 
several clinical manifestations, the most common are fatigue, loss of appetite and weight, skin lesions (mainly malar rash), arthritis, serositis (pleuritis and/or pericarditis), kidney or central nervous system involvement and hematological manifestations (cytopenias) associated with various autoantibodies, particularly antinuclear antibodies $[4,5]$.

Considering cardiovascular aspects, systemic lupus erythematosus patients have an increased risk of developing atherosclerosis, hypertension, dyslipidemia, and obesity $[6,7]$. The characteristic of dyslipidemia is the increase in total cholesterol, low-density lipoprotein (LDL), and triglyceride, with a decrease in high-density lipoprotein (HDL) [8]. The continued use of corticosteroids is a contributing factor to increased cardiovascular risk [7].

There is an increasing number of studies, still inconclusive, that evaluate the antioxidant, anti-inflammatory, and immunomodulatory effect through dietary therapy in the prevention and management of symptoms and comorbidities of patients with SLE [9].

Environmental factors can influence and modulate the onset and progression of systemic lupus erythematosus. Among those, diet plays a critical role, impacting the microbiota composition and, consequently, the immune response. There are a degree and type variation of such immune response, which depends on genetic and additional factors, such as stress, lifestyle, and medication [10]. The objective of nutritional intervention is to provide bodyweight control and a decrease in calories, saturated fat, and total fat intake. The decision to perform the present systematic review was based on the high frequency of risk factors for cardiovascular involvement in systemic lupus erythematosus patients and on the importance of analyzing and verifying the diet effect on the disease activity, as well as on the lipid parameters.

\section{Objective}

To verify the diet impact on systemic lupus erythematosus patients.

\section{Methods}

\section{Type of study}

The present study consists of a systematic review of the literature. The research question was: "What is the diet effect on systemic lupus erythematosus patients?" This study's protocol was registered on the International Prospective Register of Systematic Reviews-PROSPERO (www.crd.york.ac.uk/PROSPERO/) under number CRD42020122089.

The basic protocol was defined in April 2019, and the search strategy, eligibility criteria, bias, and outcome assessment instrument were established. Subsequently, the systematic review was registered in PROSPERO, and due to the great demand for orders and required changes, the definitive answer came only in March 2020.

\section{Inclusion criteria}

Type of patient: systemic lupus erythematosus patients, with no age and gender restriction.

Type of intervention: Calorie restricted (Low Cal), low glycaemic index (Low GI) or other diet involving the joint adequacy of these aspects.

Control: Placebo or different types of diet.

Outcome: Questionnaires and scores to evaluate the disease activity, fatigue, quality of sleep, quality of life, and lipid profile parameters.

Type of study: Randomized clinical trials.

\section{Exclusion criteria}

Studies that investigate omega-3, calcium, or any other type of supplementation were not included in this research.

\section{Searched databases}

An extensive article search was conducted in March 2021 with no language and timeframe limitations. The databases searched were: The Cochrane Central Register of Controlled Trials (CENTRAL), ClinicalTrials.gov, EMBASE, PubMed, SCOPUS, Web of Science, LILACS, and grey literature, through JSTOR, Open Grey, and Google Scholar. The keywords provided in the Medical Subject Headings (MeSH) were used to develop the primary search strategy and applied in PubMed. When words allowing different spellings were found, the synonyms appearing in $\mathrm{MeSH}$ were used. This strategy was adapted for the other databases. In LILACS, the same keywords in Spanish and English were used. See Table 1 for the applied search strategy. Manual research on relevant articles reference list was also done.

\section{Study selection}

After the search was completed, the studies found were entered on COVIDENCE (https://www.covidence.org/ home), and two independent reviewers performed the study selection (AMI and HES). The first selection filter was based on title and abstract and then on full text. There was no need for a third reviewer to resolve disagreements.

\section{Data extraction and data analyses}

For continuous data, the mean and standard deviation were extracted from the included studies. For the binary data, the number of events was extracted.

For the meta-analysis, the random-effects model would be used to estimate the effect of diet on the evaluated 
Table 1 Database search strategy

\section{Database Search (December, 2020)}

References

PubMed \#1 - ("Lupus Erythematosus, Systemic"[All Fields] OR "Systemic Lupus Erythematosus"[All Fields] OR "Lupus Erythematosus Disseminatus"[All Fields]

OR "Libman-Sacks Disease"[All Fields] OR ("lupus erythematosus, systemic"[MeSHTerms] OR ("lupus"[All Fields] AND "erythematosus"[All Fields] AND "systemic"[All Fields]) OR "systemic lupus erythematosus"[All Fields] OR ("disease"[All Fields] AND "libman"[All Fields] AND "sacks"[All Fields])) OR "Libman Sacks Disease"[All Fields] OR "Lupus Vulgaris"[All Fields] OR "Central Nervous System Lupus Vasculitis"[All Fields] OR ("lupus vasculitis, central nervous system"[MeSHTerms] OR ("lupus"[All Fields] AND "vasculitis"[All Fields] AND "central"[All Fields] AND "nervous"[All Fields] AND "system"[All Fields]) OR "central nervous system lupus vasculitis"[All Fields] OR ("systemic"[All Fields] AND "lupus"[All Fields] AND "erythematosis"[All Fields] AND "central"[All Fields] AND "nervous"[All Fields] AND "system"[All Fields])) OR "Central Nervous System Lupus"[All Fields] OR ("lupus vasculitis, central nervous system"[MeSHTerms] OR ("lupus"[All Fields] AND "vasculitis"[All Fields] AND "central"[All Fields] AND "nervous"[All Fields] AND "system"[All Fields]) OR "central nervous system lupus vasculitis"[All Fields] OR ("central"[All Fields] AND "nervous"[All Fields] AND "system"[All Fields] AND "systemic"[All Fields] AND "lupus"[All Fields] AND "erythematosis"[All Fields])) OR "Neuropsychiatric Systemic Lupus Erythematosus"[All Fields] OR "Lupus Meningoencephalitis"[All Fields] OR ("lupus vasculitis, central nervous system"[MeSHTerms] OR ("lupus"[All Fields] AND "vasculitis"[All Fields] AND "central"[All Fields] AND "nervous"[All Fields] AND "system"[All Fields]) OR "central nervous system lupus vasculitis"[All Fields] OR ("lupus"[All Fields] AND "meningoencephalitides"[All Fields])) OR ("lupus vasculitis, central nervous system"[MeSHTerms] OR ("lupus"[All Fields] AND "vasculitis"[All Fields] AND "central"[All Fields] AND "nervous"[All Fields] AND "system"[All Fields]) OR "central nervous system lupus vasculitis"[All Fields] OR ("meningoencephalitides"[All Fields] AND "lupus"[All Fields])) OR ("lupus vasculitis, central nervous system"[MeSHTerms] OR ("lupus"[All Fields] AND "vasculitis"[All Fields] AND "central"[All Fields] AND "nervous"[All Fields] AND "system"[All Fields]) OR "central nervous system lupus vasculitis"[All Fields] OR ("meningoencephalitis"[All Fields] AND "lupus"[All Fields])) OR "Lupus Panniculitis"[All Fields] OR ("panniculitis, lupus erythematosus"[MeSHTerms] OR ("panniculitis"[All Fields] AND "lupus"[All Fields] AND "erythematosus"[All Fields]) OR "lupus erythematosus panniculitis"[All Fields] OR ("lupus"[All Fields] AND "panniculitides"[All Fields])) OR ("panniculitis, lupus erythematosus"[MeSHTerms] OR ("panniculitis"[All Fields] AND "lupus"[All Fields] AND "erythematosus"[All Fields]) OR "lupus erythematosus panniculitis"[All Fields] OR ("panniculitides"[All Fields] AND "lupus"[All Fields])) OR "Panniculitis, Lupus"[All Fields] OR "Lupus Erythematosus Panniculitis"[All Fields] OR ("panniculitis, lupus erythematosus"[MeSHTerms] OR ("panniculitis"[All Fields] AND "lupus"[All Fields] AND "erythematosus"[All Fields]) OR "lupus erythematosus panniculitis"[All Fields] OR ("lupus"[All Fields] AND "erythematosus"[All Fields] AND "panniculitides"[All Fields])) OR ("panniculitis, lupus erythematosus"[MeSHTerms] OR ("panniculitis"[All Fields] AND "lupus"[All Fields] AND "erythematosus"[All Fields]) OR "lupus erythematosus panniculitis"[All Fields] OR ("panniculitides"[All Fields] AND "lupus"[All Fields] AND "erythematosus"[All Fields])) OR "Lupus Erythematosus Profundus"[All Fields] OR "Lupus Profundus"[All Fields] OR "Discoid Lupus Erythematosus"[All Fields] OR ("lupus erythematosus, discoid"[MeSHTerms] OR ("lupus"[All Fields] AND "erythematosus"[All Fields] AND "discoid"[All Fields]) OR "discoid lupus erythematosus"[All Fields] OR ("lupus"[All Fields] AND "erythematosus"[All Fields] AND "chronic"[All Fields] AND "cutaneous"[All Fields])) OR ("lupus erythematosus, discoid"[MeSHTerms] OR ("lupus"[All Fields] AND "erythematosus"[All Fields] AND "discoid"[All Fields]) OR "discoid lupus erythematosus"[All Fields] OR ("lupus"[All Fields] AND "erythematosus"[All Fields] AND "cutaneous"[All Fields] AND "chronic"[All Fields])) OR "Cutaneous Lupus Erythematosus"[All Fields] OR "Lupus Erythematosus, Subacute Cutaneous"[All Fields] OR ("lupus erythematosus, cutaneous"[MeSH Terms] OR ("lupus"[All Fields] AND "erythematosus"[All Fields] AND "cutaneous"[All Fields]) OR "cutaneous lupus erythematosus"[All Fields] OR ("lupus"[All Fields] AND "erythematosus"[All Fields] AND "cutaneous"[All Fields] AND "subacute"[All Fields]))

\#2 - ("diet"[MeSH Terms] OR "diet"[All Fields]) OR ("diet"[MeSH Terms] OR "diet"[All Fields] OR "diets"[All Fields]) OR "nutritional management"[All Fields] OR "dietary management"[All Fields] OR "Diet, Healthy"[All Fields] OR "Diets, Healthy"[All Fields] OR "Healthy Diets"[All Fields] OR "Healthy Eating"[All Fields] OR "Eating, Healthy"[All Fields] OR "Healthy Eating Index"[All Fields] OR ("healthy diet"[MeSH Terms] OR ("healthy"[All Fields] AND "diet"[All Fields]) OR "healthy diet"[All Fields] OR ("eating"[All Fields] AND "index"[All Fields] AND "healthy"[All Fields])) OR ("healthy diet"[MeSH Terms] OR ("healthy"[All Fields] AND "diet"[All Fields]) OR "healthy diet"[All Fields] OR ("eating"[All Fields] AND "indices"[All Fields] AND "healthy"[All Fields])) OR "Healthy Eating Indices"[All Fields] OR ("healthy diet"[MeSH Terms] OR ("healthy"[All Fields] AND "diet"[All Fields]) OR "healthy diet"[All Fields] OR ("index"[All Fields] AND "healthy"[All Fields] AND "eating"[All Fields])) OR ("healthy diet"[MeSHTerms] OR ("healthy"[All Fields] AND "diet"[All Fields]) OR "healthy diet"[All Fields] OR ("indices"[All Fields] AND "healthy"[All Fields] AND "eating"[All Fields])) OR ("diet, carbohydrate loading"[MeSH Terms] OR ("diet"[All Fields] AND "carbohydrate"[All Fields] AND "loading"[All Fields]) OR "carbohydrate loading diet"[All Fields] OR ("carbohydrate"[All Fields] AND "loading"[All Fields] AND "diets"[All Fields])) OR "Carbohydrate Loading Diet"[All Fields] OR "Carbohydrate Loading"[All Fields] OR ("diet, vegan"[MeSH Terms] OR ("diet"[All Fields] AND "vegan"[All Fields]) OR "vegan diet"[All Fields] OR ("diets"[All Fields] AND "vegan"[All Fields])) OR "Vegan Diets"[All Fields] OR "Vegan Diet"[All Fields] OR "Veganism"[All Fields] OR "Diet, High Protein"[All Fields] OR ("diet, high-protein"[MeSH Terms] OR ("diet"[All Fields] AND "high-protein"[All Fields]) OR "high-protein diet"[All Fields] OR ("diets"[All Fields] AND "high"[All Fields] AND "protein"[All Fields])) OR "High-Protein Diets"[All Fields] OR "High-Protein Diet"[All Fields] OR "High Protein Diet"[All Fields] OR "Diet, High Protein Low Carbohydrate"[All Fields] OR ("diet, high-protein low-carbohydrate"[MeSH Terms] OR ("diet"[All Fields] AND "high-protein"[All Fields] AND "low-carbohydrate"[All Fields]) OR "high-protein low-carbohydrate diet"[All Fields] OR ("diets"[All Fields] AND "high"[All Fields] AND "protein"[All Fields] AND "low"[All Fields] AND "carbohydrate"[All Fields])) OR ("diet, high-protein lowcarbohydrate"[MeSHTerms] OR ("diet"[All Fields] AND "high-protein"[All Fields] AND "low-carbohydrate"[All Fields]) OR "high-protein low-carbohydrate diet"[All Fields] OR ("low"[All Fields] AND "carbohydrate"[All Fields] AND "diet"[All Fields] AND "high"[All Fields] AND "protein"[All Fields])) OR ("diet, high-protein low-carbohydrate"[MeSH Terms] OR ("diet"[All Fields] AND "high-protein"[All Fields] AND "low-carbohydrate"[All Fields]) OR "high-protein low-carbohydrate diet"[All Fields] OR ("low"[All Fields] AND "carbohydrate"[All Fields] AND "diets"[All Fields] AND "high"[All Fields] AND "protein"[All Fields])) OR "Carbohydrate-Restricted High-Protein Diet"[All Fields] OR "Carbohydrate Restricted High Protein Diet"[All Fields] OR ("diet, high-protein lowcarbohydrate"[MeSH Terms] OR ("diet"[All Fields] AND "high-protein"[All Fields] AND "low-carbohydrate"[All Fields]) OR "high-protein low-carbohydrate diet"[All Fields] OR ("diet"[All Fields] AND "carbohydrate"[All Fields] AND "restricted"[All Fields] AND "high"[All Fields] AND "protein"[All Fields])) OR ("diet, high-protein low-carbohydrate"[MeSHTerms] OR ("diet"[All Fields] AND "high-protein"[All Fields] AND "low-carbohydrate"[All Fields]) OR "high-protein low-carbohydrate diet"[All Fields] OR ("diets"[All Fields] AND "carbohydrate"[All Fields] AND "restricted"[All Fields] AND "high"[All Fields] AND "protein"[All Fields])) OR ("diet, high-protein low-carbohydrate"[MeSHTerms] OR ("diet"[All Fields] AND "high-protein"[All Fields] AND "low-carbohydrate"[All Fields]) OR "high-protein low-carbohydrate diet"[All Fields] OR ("high"[All Fields] AND "protein"[All Fields] AND "diet"[All Fields] AND "carbohydrate"[All Fields] AND "restricted"[All Fields])) OR ("diet, high-protein low-carbohydrate"[MeSH Terms] OR ("diet"[All Fields] AND "high-protein"[All Fields] AND "lowcarbohydrate"[All Fields]) OR "high-protein low-carbohydrate diet"[All Fields] OR ("high"[All Fields] AND "protein"[All Fields] AND "diets"[All Fields] AND "carbohydrate"[All Fields] AND "restricted"[All Fields])) OR ("diet, high-protein low-carbohydrate"[MeSHTerms] OR ("diet"[All Fields] AND "high-protein"[All Fields] AND "low-carbohydrate"[All Fields]) OR "high-protein low-carbohydrate diet"[All Fields] OR ("carbohydrate"[All Fields] AND "restricted"[All Fields] AND "high"[All Fields] AND "protein"[All Fields] AND "diets"[All Fields])) OR ("diet, high-protein low-carbohydrate"[MeSH Terms] OR ("diet"[All Fields] AND "high-protein"[All Fields] AND "low-carbohydrate"[All Fields]) OR "high-protein low-carbohydrate diet"[All Fields] OR ("carbohydrate"[All Fields] AND "restricted"[All Fields] AND "high"[All Fields] AND "protein"[All Fields] AND "diets"[All Fields])) OR "Low-Carbohydrate High-Protein Diets"[All Fields] OR "Low Carbohydrate High Protein Diets"[All Fields] OR "High-Protein Low-Carbohydrate Diet"[All Fields] OR "High Protein LowCarbohydrate Diet"[All Fields] OR "High-Protein Low-Carbohydrate Diets"[All Fields] OR "High Protein Low Carbohydrate Diets"[All Fields] OR "Low-Carbohydrate High-Protein Diet"[All Fields] OR ("diet, high-protein low-carbohydrate"[MeSHTerms] OR ("diet"[All Fields] AND "high-protein"[All Fields] AND "low-carbohydrate"[All Fields]) OR "high-protein low-carbohydrate diet"[All Fields] OR ("diet"[All Fields] 
Table 1 (continued)

AND"low"[All Fields] AND"carbohydrate"[All Fields] AND"high"[All Fields] AND "protein"[All Fields])) OR ("diet, high-protein low-carbohydrate"[MeSH Terms] OR ("diet"[All Fields] AND "high-protein"[All Fields] AND"low-carbohydrate"[All Fields]) OR"high-protein low-carbohydrate diet"[All Fields] OR ("diets"[All Fields] AND"low"[All Fields] AND"carbohydrate"[All Fields] AND “high"[All Fields] AND "protein"[All Fields])) OR ("diet, high-protein low-carbohydrate"[MeSH Terms] OR ("diet"[All Fields] AND"high-protein"[All Fields] AND"low-carbohydrate"[All Fields]) OR"high-protein lowcarbohydrate diet"[All Fields] OR ("high"[All Fields] AND "protein"[All Fields] AND "diet"[All Fields] AND"low"[All Fields] AND"carbohydrate"[All Fields])) OR ("diet, high-protein low-carbohydrate"[MeSHTerms] OR ("diet"[All Fields] AND"high-protein"[All Fields] AND"low-carbohydrate"[All Fields]) OR "high-protein low-carbohydrate diet"[All Fields] OR ("high"[All Fields] AND"protein"[All Fields] AND"diets"[All Fields] AND"low"[All Fields] AND "carbohydrate"[All Fields])) OR"Low Carbohydrate High Protein Diet"[All Fields] OR ("diet, high-protein low-carbohydrate"[MeSHTerms] OR ("diet"[All Fields] AND "high-protein"[All Fields] AND"low-carbohydrate"[All Fields]) OR"high-protein low-carbohydrate diet"[All Fields] OR ("high"[All Fields] AND "protein"[All Fields] AND"carbohydrate"[All Fields] AND"restricted"[All Fields] AND "diets"[All Fields])) OR ("diet, high-protein low-carbohydrate"[MeSH Terms] OR ("diet"[All Fields] AND "high-protein"[All Fields] AND"low-carbohydrate"[All Fields]) OR"high-protein low-carbohydrate diet"[All Fields] OR ("carbohydrate"[All Fields] AND"restricted"[All Fields] AND "diet"[All Fields] AND"high"[All Fields] AND"protein"[All Fields])) OR ("diet, high-protein low-carbohydrate"[MeSHTerms] OR ("diet"[All Fields] AND"high-protein"[All Fields] AND"low-carbohydrate"[All Fields]) OR"high-protein lowcarbohydrate diet"[All Fields] OR ("carbohydrate"[All Fields] AND"restricted"[All Fields] AND"diets"[All Fields] AND"high"[All Fields] AND"protein"[All Fields])) OR ("diet, high-protein low-carbohydrate"[MeSH Terms] OR ("diet"[All Fields] AND"high-protein"[All Fields] AND"low-carbohydrate"[All Fields]) OR"high-protein low-carbohydrate diet"[All Fields] OR ("diet"[All Fields] AND"high"[All Fields] AND"protein"[All Fields] AND"carbohydrate"[All Fields] AND"restricted"[All Fields])) OR ("diet, high-protein low-carbohydrate"[MeSH Terms] OR ("diet"[All Fields] AND"high-protein"[All Fields] AND"lowcarbohydrate"[All Fields]) OR "high-protein low-carbohydrate diet"[All Fields] OR ("diets"[All Fields] AND"high"[All Fields] AND"protein"[All Fields] AND "carbohydrate"[All Fields] AND"restricted"[All Fields])) OR ("diet, high-protein low-carbohydrate"[MeSHTerms] OR ("diet"[All Fields] AND"high-protein"[All Fields] AND"low-carbohydrate"[All Fields]) OR"high-protein low-carbohydrate diet"[All Fields] OR ("high"[All Fields] AND "protein"[All Fields] AND"carbohydrate"[All Fields] AND"restricted"[All Fields] AND"diets"[All Fields])) OR ("diet, high-protein low-carbohydrate"[MeSH Terms] OR ("diet"[All Fields] AND"high-protein"[All Fields] AND"low-carbohydrate"[All Fields]) OR"high-protein low-carbohydrate diet"[All Fields] OR ("high"[All Fields] AND"protein"[All Fields] AND "carbohydrate"[All Fields] AND"restricted"[All Fields] AND"diet"[All Fields])) OR ("diet, high-protein low-carbohydrate"[MeSH Terms] OR ("diet"[All Fields] AND"high-protein"[All Fields] AND"low-carbohydrate"[All Fields]) OR"high-protein lowcarbohydrate diet"[All Fields] OR ("high"[All Fields] AND "protein"[All Fields] AND"carbohydrate"[All Fields] AND "restricted"[All Fields] AND"diet"[All Fields])) OR "South Beach Diet"[All Fields] OR ("diet, high-protein low-carbohydrate"[MeSH Terms] OR ("diet"[All Fields] AND"high-protein"[All Fields] AND"low-carbohydrate"[All Fields]) OR"high-protein low-carbohydrate diet"[All Fields] OR ("diet"[All Fields] AND"south"[All Fields] AND"beach"[All Fields])) OR "Atkins Diet"[All Fields] OR"Diet, Atkins"[All Fields] OR"Diabetic Diets"[All Fields] OR ("diet, diabetic"[MeSH Terms] OR ("diet"[All Fields] AND "diabetic"[All Fields]) OR"diabetic diet"[All Fields] OR ("diets"[All Fields] AND "diabetic"[All Fields])) OR"Diabetic Diet"[All Fields] OR"Therapy, Diet"[All Fields] OR "Diet Therapies"[All Fields] OR ("diet therapy"[MeSH Terms] OR ("diet"[All Fields] AND"therapy"[All Fields]) OR "diet therapy"[All Fields] OR ("therapies"[All Fields] AND "diet"[All Fields])) OR "Dietary Modification"[All Fields] OR "Dietary Modifications"[All Fields] OR ("diet therapy"[MeSH Terms] OR ("diet"[All Fields] AND"therapy"[All Fields]) OR "diet therapy"[All Fields] OR ("modification"[All Fields] AND"dietary"[All Fields])) OR ("diet therapy"[MeSHTerms] OR ("diet"[All Fields] AND"therapy"[All Fields]) OR "diet therapy"[All Fields] OR ("modifications"[All Fields] AND"dietary"[All Fields])) OR "Diet Modification"[All Fields] OR"Diet Modifications"[All Fields] OR"Modification, Diet"[All Fields] OR "Modifications, Diet"[All Fields] OR "Atherogenic Diet"[All Fields] OR “Atherogenic Diets"[All Fields] OR ("diet, atherogenic"[MeSH Terms] OR ("diet"[All Fields] AND"atherogenic"[All Fields]) OR "atherogenic diet"[All Fields] OR ("diets"[All Fields] AND "atherogenic"[All Fields])) OR ("diet, vegetarian"[MeSH Terms] OR ("diet"[All Fields] AND "vegetarian"[All Fields]) OR "vegetarian diet"[All Fields] OR ("diets"[All Fields] AND"vegetarian"[All Fields])) OR "Vegetarian Diets"[All Fields] OR "Vegetarian Diet"[All Fields] OR"Vegetarianism"[All Fields] OR ("diet, macrobiotic"[MeSH Terms] OR ("diet"[All Fields] AND"macrobiotic"[All Fields]) OR "macrobiotic diet"[All Fields] OR ("diets"[All Fields] AND"macrobiotic"[All Fields])) OR"Macrobiotic Diet"[All Fields] OR"Macrobiotic Diets"[All Fields] OR "Diet, Fat Restricted"[All Fields] OR "Fat-Restricted Diet"[All Fields] OR ("diet, fat-restricted"[MeSH Terms] OR ("diet"[All Fields] AND"fat-restricted"[Al Fields]) OR "fat-restricted diet"[All Fields] OR ("diets"[All Fields] AND"fat"[All Fields] AND "restricted"[All Fields])) OR "Fat Restricted Diet"[All Fields] OR "Fat-Restricted Diets"[All Fields] OR "Diet, Low-Fat"[All Fields] OR"Diet, Low Fat"[All Fields] OR ("diet, fat-restricted"[MeSH Terms] OR ("diet"[All Fields] AND "fat-restricted"[All Fields]) OR "fat-restricted diet"[All Fields] OR ("diets"[All Fields] AND"low"[All Fields] AND"fat"[All Fields])) OR"Low-Fat Diet"[All Fields] OR"Low-Fat Diets"[All Fields] OR ("diet, fat-restricted"[MeSH Terms] OR ("diet"[All Fields] AND "fat-restricted"[All Fields]) OR"fat-restricted diet"[All Fields] OR ("diet"[All Fields] AND"fat"[All Fields] AND"free"[All Fields])) OR ("diet, fatrestricted"[MeSHTerms] OR ("diet"[All Fields] AND "fat-restricted"[All Fields]) OR "fat-restricted diet"[All Fields] OR ("diet"[All Fields] AND"fat"[All Fields] AND"free"[All Fields])) OR ("diet, fat-restricted"[MeSH Terms] OR ("diet"[All Fields] AND "fat-restricted"[All Fields]) OR "fat-restricted diet"[All Fields] OR ("diets"[All Fields] AND "fat"[All Fields] AND"free"[All Fields])) OR"Fat-Free Diet"[All Fields] OR "Fat-Free Diets"[All Fields] OR"Diet, Protein Restricted"[All Fields] OR"Low-Protein Diet"[All Fields] OR"Low Protein Diet"[All Fields] OR"Protein-Restricted Diet"[All Fields] OR ("diet, protein-restricted"[MeSH Terms] OR ("diet"[All Fields] AND "protein-restricted"[All Fields]) OR"protein-restricted diet"[All Fields] OR ("diets"[All Fields] AND "protein"[All Fields] AND"restricted"[All Fields])) OR "Protein Restricted Diet"[All Fields] OR "Protein-Restricted Diets"[All Fields] OR ("diet, protein-restricted"[MeSH Terms] OR ("diet"[All Fields] AND "protein-restricted"[All Fields]) OR"protein-restricted diet"[All Fields] OR ("diet"[All Fields] AND"low"[All Fields] AND "protein"[All Fields])) OR ("diet, protein-restricted"[MeSH Terms] OR ("diet"[All Fields] AND "protein-restricted"[All Fields]) OR"protein-restricted diet"[All Fields] OR ("diet"[All Fields] AND"low"[All Fields] AND "protein"[All Fields])) OR ("diet, protein-restricted"[MeSH Terms] OR ("diet"[All Fields] AND "protein-restricted"[All Fields]) OR"protein-restricted diet"[All Fields] OR ("diets"[All Fields] AND"low"[All Fields] AND"protein"[All Fields])) OR "Low-Protein Diets"[All Fields] OR ("diet, protein-restricted"[MeSHTerms] OR ("diet"[All Fields] AND "protein-restricted"[All Fields]) OR"protein-restricted diet"[All Fields] OR ("diet"[All Fields] AND "protein"[All Fields] AND "free"[All Fields])) OR ("diet, protein-restricted"[MeSH Terms] OR ("diet"[All Fields] AND "protein-restricted"[All Fields]) OR"protein-restricted diet"[All Fields] OR ("diet"[All Fields] AND"protein"[All Fields] AND"free"[All Fields])) OR ("diet, protein-restricted"[MeSHTerms] OR ("diet"[All Fields] AND "protein-restricted"[All Fields]) OR"protein-restricted diet"[All Fields] OR ("diets"[All Fields] AND "protein"[All Fields] AND"free"[All Fields])) OR "Protein-Free Diet"[All Fields] OR"Protein-Free Diets"[All Fields] OR"Mediterranean Diet"[All Fields] OR ("diet, mediterranean"[MeSH Terms] OR ("diet"[All Fields] AND"mediterranean"[All Fields]) OR"mediterranean diet"[All Fields] OR ("diets"[All Fields] AND "mediterranean"[All Fields])) OR"Mediterranean Diets"[All Fields] OR "Diet, Carbohydrate Restricted"[All Fields] OR ("diet, carbohydraterestricted"[MeSH Terms] OR ("diet"[All Fields] AND"carbohydrate-restricted"[All Fields]) OR"carbohydrate-restricted diet"[All Fields] OR ("diet"[All Fields] AND"low"[All Fields] 
Table 1 (continued)

Database Search (December, 2020)

References

AND"carbohydrate"[All Fields])) OR ("diet, carbohydrate-restricted"[MeSHTerms] OR ("diet"[All Fields] AND"carbohydrate-restricted"[All Fields]) OR"carbohydrate-restricted diet"[All Fields] OR ("carbohydrate"[All Fields] AND"diet"[All Fields] AND"low"[All Fields])) OR ("diet, carbohydraterestricted"[MeSH Terms] OR ("diet"[All Fields] AND "carbohydrate-restricted"[All Fields]) OR"carbohydrate-restricted diet"[All Fields] OR ("carbohydrate"[All Fields] AND "diets"[All Fields] AND"low"[All Fields])) OR ("diet, carbohydrate-restricted"[MeSH Terms] OR ("diet"[All Fields] AND "carbohydrate-restricted"[All Fields]) OR "carbohydrate-restricted diet"[All Fields] OR ("diets"[All Fields] AND"low"[All Fields] AND"carbohydrate"[All Fields])) OR “Low Carbohydrate Diets"[All Fields] OR "Carbohydrate-Restricted Diet"[All Fields] OR "Carbohydrate Restricted Diet"[All Fields] OR"Carbohydrate-Restricted Diets"[All Fields] OR ("diet, carbohydrate-restricted"[MeSHTerms] OR ("diet"[All Fields] AND "carbohydrate-restricted"[All Fields]) OR"carbohydrate-restricted diet"[All Fields] OR ("diets"[All Fields] AND "carbohydrate"[All Fields] AND"restricted"[All Fields])) OR"Low-Carbohydrate Diet"[All Fields] OR ("diet, carbohydrate-restricted"[MeSH Terms] OR ("diet"[All Fields] AND"carbohydrate-restricted"[All Fields]) OR"carbohydraterestricted diet"[All Fields] OR ("diet"[All Fields] AND"low"[All Fields] AND"carbohydrate"[All Fields])) OR ("diet, carbohydrate-restricted"[MeSH Terms] OR ("diet"[All Fields] AND"carbohydrate-restricted"[All Fields]) OR"carbohydrate-restricted diet"[All Fields] OR ("diets"[All Fields] AND"low"[All Fields] AND"carbohydrate"[All Fields])) OR "Low Carbohydrate Diet"[All Fields] OR"Low-Carbohydrate Diets"[All Fields] OR "Diet, Gluten Free"[All Fields] OR "Gluten-Free Diet"[All Fields] OR ("diet, gluten-free"[MeSHTerms] OR ("diet"[All Fields] AND"gluten-free"[All Fields]) OR "gluten-free diet"[All Fields] OR ("diets"[All Fields] AND "gluten"[All Fields] AND "free"[All Fields])) OR "Gluten Free Diet"[All Fields] OR "Gluten-Free Diets"[All Fields] OR"Ketogenic Diet"[All Fields] OR ("diet, ketogenic"[MeSH Terms] OR ("diet"[All Fields] AND "ketogenic"[All Fields]) OR "ketogenic diet"[All Fields] OR ("diets"[All Fields] AND "ketogenic"[All Fields])) OR"Ketogenic Diets"[All Fields] OR ("diet, high-fat"[MeSHTerms] OR ("diet"[All Fields] AND"high-fat"[All Fields]) OR "high-fat diet"[All Fields] OR ("diets"[All Fields] AND"high"[All Fields] AND"fat"[All Fields])) OR"High-Fat Diet"[All Fields] OR"High-Fat Diets"[All Fields] OR "Diet, High Fat"[All Fields] OR ("diet, high-fat"[MeSH Terms] OR ("diet"[All Fields] AND"high-fat"[All Fields]) OR "high-fat diet"[All Fields] OR ("diets"[All Fields] AND "high"[All Fields] AND"fat"[All Fields])) OR"High Fat Diet"[All Fields] OR"High Fat Diets"[All Fields] OR “Paleolithic Diet"[All Fields] OR ("diet, paleolithic"[MeSH Terms] OR ("diet"[All Fields] AND "paleolithic"[All Fields]) OR"paleolithic diet"[All Fields] OR ("diet"[All Fields] AND"stone"[All Fields] AND "age"[All Fields])) OR ("diet, paleolithic"[MeSH Terms] OR ("diet"[All Fields] AND "paleolithic"[All Fields]) OR"paleolithic diet"[All Fields] OR ("diets"[All Fields] AND "stone"[All Fields] AND "age"[All Fields])) OR "Stone Age Diet"[All Fields] OR "Stone Age Diets"[All Fields] OR ("diet, paleolithic"[MeSH Terms] OR ("diet"[All Fields] AND "paleolithic"[All Fields]) OR"paleolithic diet"[All Fields] OR ("diet"[All Fields] AND"paleo"[All Fields])) OR ("diet, paleolithic"[MeSH Terms] OR ("diet"[All Fields] AND"paleolithic"[All Fields]) OR"paleolithic diet"[All Fields] OR ("diets"[All Fields] AND "paleo"[All Fields])) OR"Paleo Diet"[All Fields] OR"Paleo Diets"[All Fields] OR ("diet, paleolithic"[MeSH Terms] OR ("diet"[All Fields]

AND"paleolithic"[All Fields]) OR "paleolithic diet"[All Fields] OR ("diet"[All Fields] AND"caveman"[All Fields])) OR ("diet, paleolithic"[MeSH Terms] OR ("diet"[All Fields] AND"paleolithic"[All Fields]) OR"paleolithic diet"[All Fields] OR ("caveman"[All Fields] AND "diet"[All Fields])) OR ("diet, paleolithic"[MeSH Terms] OR ("diet"[All Fields] AND"paleolithic"[All Fields]) OR "paleolithic diet"[All Fields] OR ("caveman"[All Fields] AND "diets"[All Fields])) OR ("diet, paleolithic"[MeSH Terms] OR ("diet"[All Fields] AND "paleolithic"[All Fields]) OR"paleolithic diet"[All Fields] OR ("diets"[All Fields] AND "caveman"[All Fields])) OR ("diet, paleolithic"[MeSH Terms] OR ("diet"[All Fields] AND "paleolithic"[All Fields]) OR"paleolithic diet"[All Fields] OR ("diet"[All Fields] AND"hunter"[All Fields] AND"gatherer"[All Fields])) OR ("diet, paleolithic"[MeSHTerms] OR ("diet"[All Fields] AND "paleolithic"[All Fields]) OR "paleolithic diet"[All Fields] OR ("diet"[All Fields] AND "hunter"[All Fields] AND"gatherer"[All Fields])) OR ("diet, paleolithic"[MeSHTerms] OR ("diet"[All Fields] AND"paleolithic"[All Fields]) OR"paleolithic diet"[All Fields] OR ("diets"[All Fields] AND"hunter"[All Fields] AND"gatherer"[All Fields])) OR"HunterGatherer Diet"[All Fields] OR"Hunter-Gatherer Diets"[All Fields] OR ("diet, western"[MeSH Terms] OR ("diet"[All Fields] AND"western"[All Fields]) OR "western diet"[All Fields] OR ("diets"[All Fields] AND "western"[All Fields])) OR"Western Diets"[All Fields] OR"Western Dietary Pattern"[All Fields] OR ("diet, western"[MeSHTerms] OR ("diet"[All Fields] AND"western"[All Fields]) OR "western diet"[All Fields] OR ("dietary"[All Fields] AND"pattern"[All Fields] AND "western"[All Fields])) OR ("diet, western"[MeSH Terms] OR ("diet"[All Fields] AND "western"[All Fields]) OR "western diet"[All Fields] OR ("dietary"[All Fields] AND"patterns"[All Fields] AND "western"[All Fields])) OR ("diet, western"[MeSH Terms] OR ("diet"[All Fields] AND"western"[All Fields]) OR"western diet"[All Fields] OR ("pattern"[All Fields] AND "western"[All Fields] AND "dietary"[All Fields])) OR ("diet, western"[MeSH Terms] OR ("diet"[All Fields] AND "western"[All Fields]) OR"western diet"[All Fields] OR ("patterns"[All Fields] AND "western"[All Fields] AND"dietary"[All Fields])) OR"Western Dietary Patterns"[All Fields] OR ("diet, western"[MeSH Terms] OR ("diet"[All Fields] AND "western"[All Fields]) OR"western diet"[All Fields] OR ("diet"[All Fields] AND"occidental"[All Fields])) OR ("diet, western"[MeSHTerms] OR ("diet"[All Fields] AND "western"[All Fields]) OR"western diet"[All Fields] OR ("diets"[All Fields] AND"occidental"[All Fields])) OR "Occidental Diet"[All Fields] OR "Occidental Diets"[All Fields] OR "Western Diet"[All Fields] OR ("diet, western"[MeSH Terms] OR ("diet"[All Fields] AND "western"[All Fields]) OR "western diet"[All Fields] OR ("meat"[All Fields] AND "sweet"[All Fields] AND "diet"[All Fields])) OR ("diet, western"[MeSHTerms] OR ("diet"[All Fields] AND "western"[All Fields]) OR "western diet"[All Fields] OR ("diet"[All Fields] AND"meat"[All Fields] AND"sweet"[All Fields])) OR ("diet, western"[MeSH Terms] OR ("diet"[All Fields] AND "western"[All Fields]) OR"western diet"[All Fields] OR ("diets"[All Fields] AND"meat"[All Fields] AND "sweet"[All Fields])) OR ("diet, western"[MeSH Terms] OR ("diet"[All Fields] AND "western"[All Fields]) OR"western diet"[All Fields] OR ("meat"[All Fields] AND "sweet"[All Fields] AND "diet"[All Fields])) OR ("diet, western"[MeSH Terms] OR ("diet"[All Fields] AND"western"[All Fields]) OR"western diet"[All Fields] OR ("meat"[All Fields] AND"sweet"[All Fields] AND"diets"[All Fields])) \#3 - ("Clinical Trials, Randomized"[All Fields] OR "Trials, Randomized Clinical"[All Fields] OR "Controlled Clinical Trials, Randomized"[All Fields OR "Randomized Controlled Trial"[All Fields]) \#4 - (\#1 AND \#2 AND \#3) 
Table 1 (continued)

\begin{tabular}{|c|c|c|}
\hline Database & Search (December, 2020) & References \\
\hline Scopus & 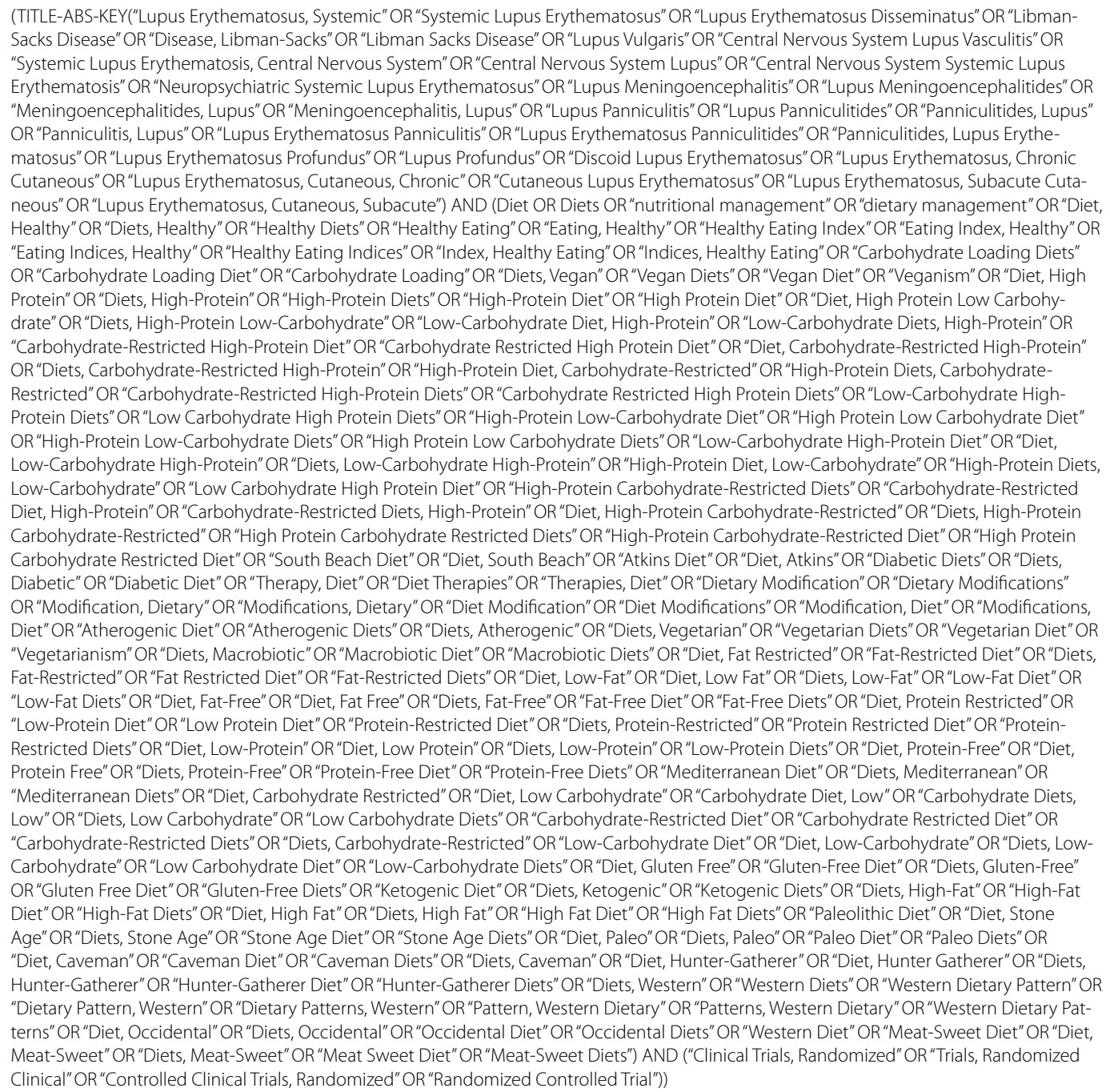 & 400 \\
\hline
\end{tabular}


Table 1 (continued)

\begin{tabular}{|c|c|c|}
\hline Database & Search (December, 2020) & References \\
\hline $\begin{array}{l}\text { Web of } \\
\text { Science }\end{array}$ & 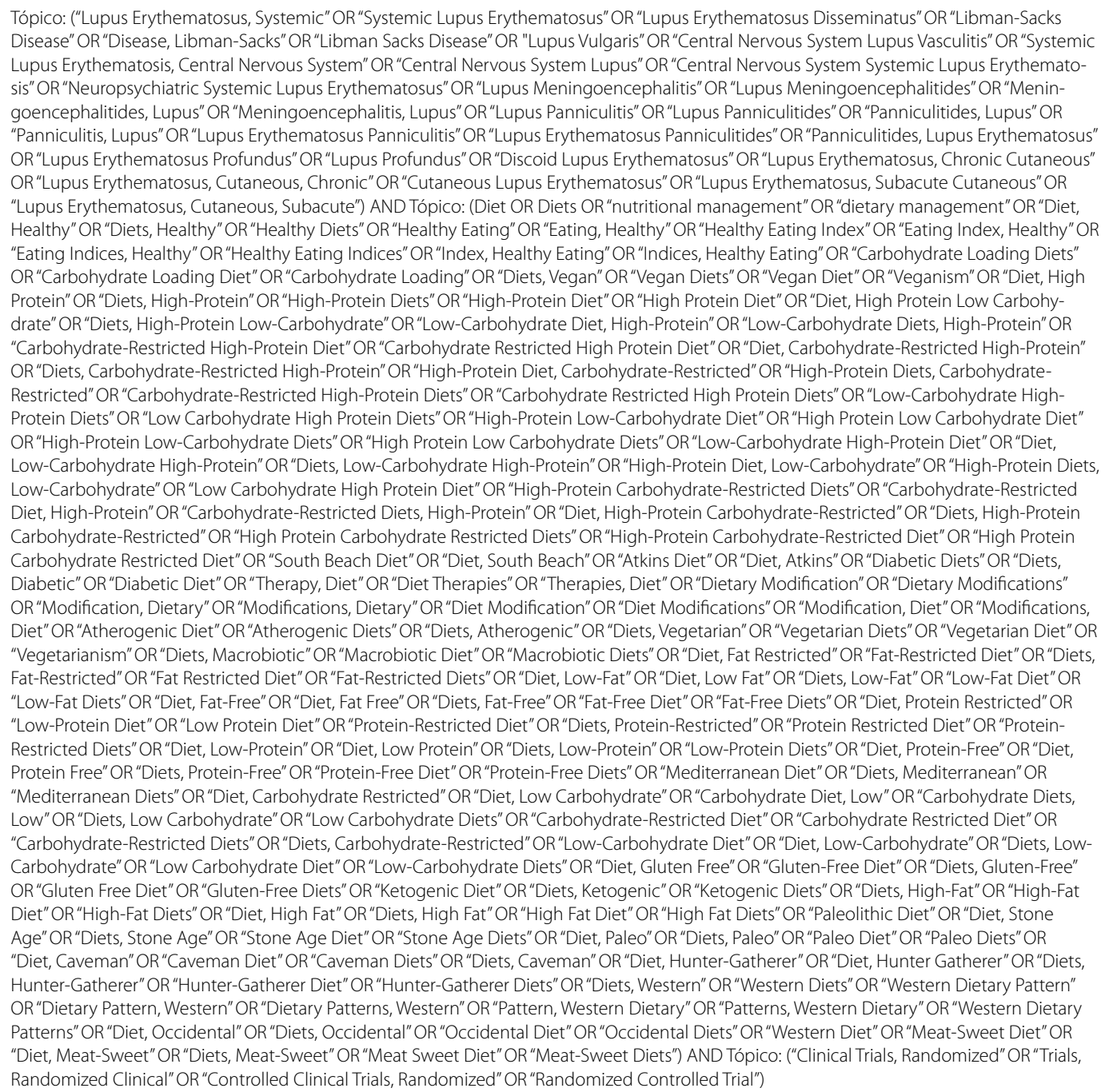 & 05 \\
\hline
\end{tabular}


Table 1 (continued)

\begin{tabular}{|c|c|c|}
\hline Database & Search (December, 2020) & References \\
\hline Cochrane & 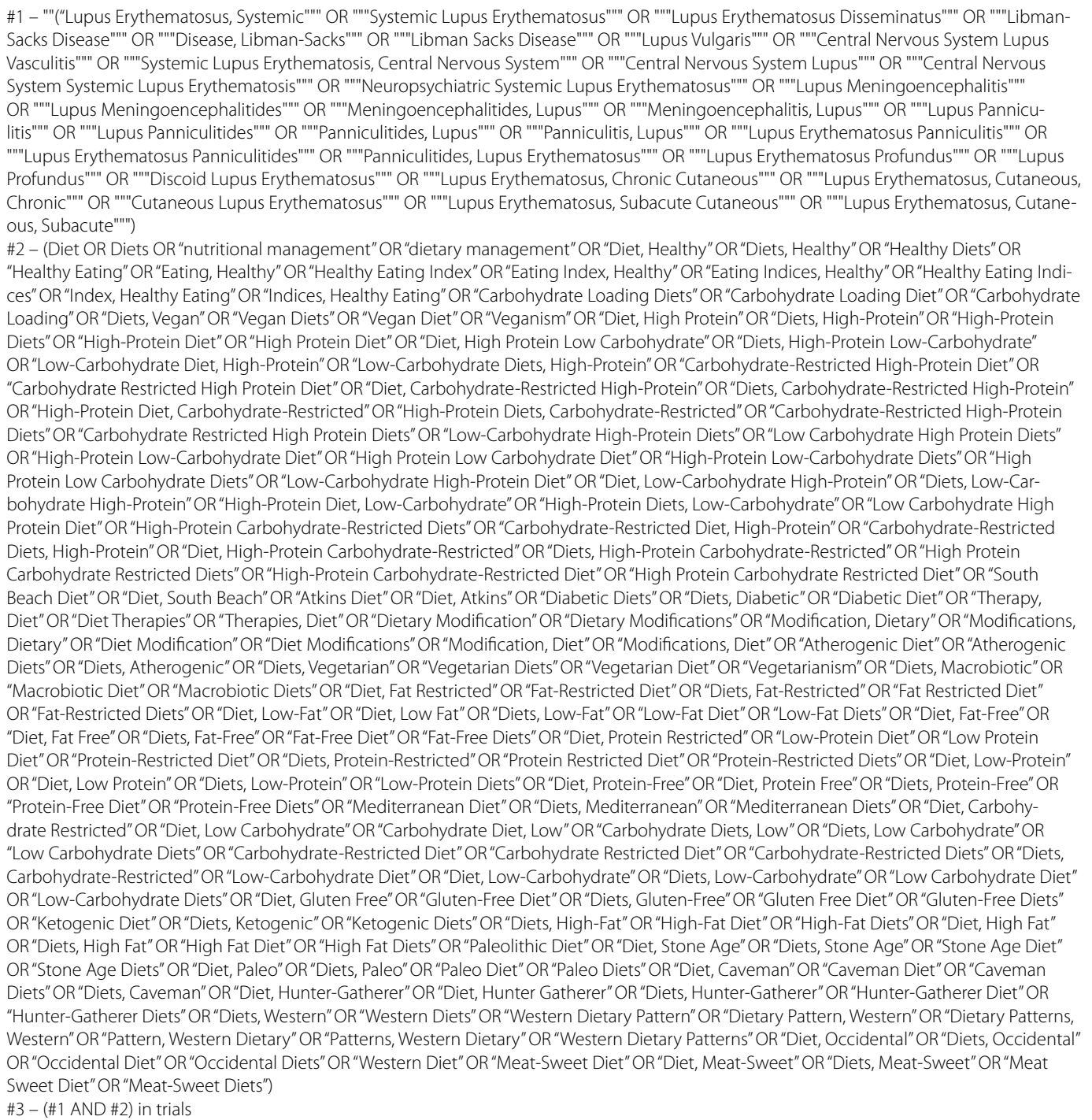 & 29 \\
\hline
\end{tabular}


Table 1 (continued)

\section{Database Search (December, 2020)}

ClinicalTri- \#1 - "'("Lupus Erythematosus, Systemic"'" OR "'"'Systemic Lupus Erythematosus"'"' OR "'"'Lupus Erythematosus Disseminatus"'" OR "'"'Libman-

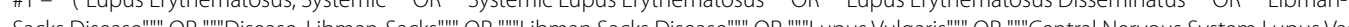
Sacks Disease""'" OR "'"'Disease, Libman-Sacks"'"' OR "'"'Libman Sacks Disease"'"' OR "'"'Lupus Vulgaris"'"' OR "'"'Central Nervous System Lupus Vas-
culitis"'"' OR "'"'Systemic Lupus Erythematosis, Central Nervous System"'"' OR "'"'Central Nervous System Lupus"'"' OR "'"'Central Nervous System Systemic Lupus Erythematosis"'"' OR "'"'Neuropsychiatric Systemic Lupus Erythematosus"'"' OR "'"'Lupus Meningoencephalitis"'"' OR "'"'Lupus Meningoencephalitides"'"' OR "'"'Meningoencephalitides, Lupus"'"' OR "'"'Meningoencephalitis, Lupus"'"' OR "'"'Lupus Panniculitis"'"' OR "'"'Lupus Panniculitides"'"' OR "'"'Panniculitides, Lupus"'"' OR "'"'Panniculitis, Lupus"'"' OR "'"'Lupus Erythematosus Panniculitis"'"' OR "'"'Lupus Erythematosus Panniculitides"'"' OR "'"'Panniculitides, Lupus Erythematosus"'" OR "'"'Lupus Erythematosus Profundus"'"' OR "'"'Lupus Profundus"'" OR "'"'Discoid Lupus Erythematosus"'"' OR "'"'Lupus Erythematosus, Chronic Cutaneous"'"' OR "'"'Lupus Erythematosus, Cutaneous, Chronic"'"' OR "'"'Cutaneous Lupus Erythematosus"'"' OR "'"'Lupus Erythematosus, Subacute Cutaneous"'"' OR "'"'Lupus Erythematosus, Cutaneous, Subacute""'"') \#2 - (Diet OR Diets OR"nutritional management"OR "dietary management"OR "Diet, Healthy"OR "Diets, Healthy"OR "Healthy Diets" OR"Healthy Eating" OR "Eating, Healthy" OR "Healthy Eating Index"OR "Eating Index, Healthy"OR"Eating Indices, Healthy"OR"Healthy Eating Indices" OR"Index, Healthy Eating"OR "Indices, Healthy Eating"OR "Carbohydrate Loading Diets"OR "Carbohydrate Loading Diet" OR "Carbohydrate Loading"OR "Diets, Vegan" OR "Vegan Diets" OR"Vegan Diet"OR"Veganism"OR"Diet, High Protein"OR"Diets, High-Protein"OR"High-Protein Diets"OR"High-Protein Diet"OR"High Protein Diet" OR "Diet, High Protein Low Carbohydrate"OR "Diets, High-Protein Low-Carbohydrate" OR "Low-Carbohydrate Diet, High-Protein" OR "LowCarbohydrate Diets, High-Protein" OR"Carbohydrate-Restricted High-Protein Diet"OR"Carbohydrate Restricted High Protein Diet"OR"Diet, Carbohydrate-Restricted High-Protein" OR "Diets, Carbohydrate-Restricted High-Protein" OR"High-Protein Diet, Carbohydrate-Restricted"OR"High-Protein Diets, Carbohydrate-Restricted" OR "Carbohydrate-Restricted High-Protein Diets"OR "Carbohydrate Restricted High Protein Diets" OR"Low-Carbohydrate High-Protein Diets" OR “Low Carbohydrate High Protein Diets" OR "High-Protein Low-Carbohydrate Diet"OR "High Protein Low Carbohydrate Diet"OR "High-Protein Low-Carbohydrate Diets"OR"High Protein Low Carbohydrate Diets"OR"Low-Carbohydrate High-Protein Diet" OR"Diet, Low-Carbohydrate High-Protein" OR "Diets, Low-Carbohydrate High-Protein"OR "High-Protein Diet, Low-Carbohydrate"OR "High-Protein Diets, Low-Carbohydrate" OR "Low Carbohydrate High Protein Diet" OR "High-Protein Carbohydrate-Restricted Diets" OR "Carbohydrate-Restricted Diet, High-Protein"OR "Carbohydrate-Restricted Diets, High-Protein" OR "Diet, High-Protein Carbohydrate-Restricted"OR "Diets, High-Protein Carbohydrate-Restricted" OR "High Protein Carbohydrate Restricted Diets" OR"High-Protein Carbohydrate-Restricted Diet" OR "High Protein Carbohydrate Restricted Diet" OR"South Beach Diet" OR"Diet, South Beach" OR"Atkins Diet"OR"Diet, Atkins"OR"Diabetic Diets"OR "Diets, Diabetic"OR "Diabetic Diet" OR"Therapy, Diet" OR "Diet Therapies"OR "Therapies, Diet"OR"Dietary Modification"OR "Dietary Modifications"OR"Modification, Dietary" OR "Modifications, Dietary"OR "Diet Modification" OR "Diet Modifications" OR"Modification, Diet"OR"Modifications, Diet" OR "Atherogenic Diet" OR "Atherogenic Diets" OR "Diets, Atherogenic"OR "Diets, Vegetarian" OR"Vegetarian Diets" OR"Vegetarian Diet" OR "Vegetarianism"OR "Diets, Macrobiotic"OR"Macrobiotic Diet" OR"Macrobiotic Diets" OR "Diet, Fat Restricted" OR"Fat-Restricted Diet"OR "Diets, Fat-Restricted"OR "Fat Restricted Diet"OR "Fat-Restricted Diets" OR "Diet, Low-Fat"OR"Diet, Low Fat"OR"Diets, Low-Fat"OR "Low-Fat Diet"OR"Low-Fat Diets"OR"Diet, Fat-Free"OR"Diet, Fat Free" OR "Diets, Fat-Free"OR "Fat-Free Diet"OR"Fat-Free Diets" OR "Diet, Protein Restricted"OR"Low-Protein Diet"OR "Low Protein Diet" OR"Protein-Restricted Diet"OR"Diets, Protein-Restricted"OR"Protein Restricted Diet" OR "Protein-Restricted Diets"OR "Diet, Low-Protein"OR “Diet, Low Protein"OR"Diets, Low-Protein" OR"Low-Protein Diets" OR"Diet, Protein-Free"OR "Diet, Protein Free" OR"Diets, Protein-Free" OR "Protein-Free Diet" OR"Protein-Free Diets" OR"Mediterranean Diet"OR"Diets, Mediterranean" OR"Mediterranean Diets" OR "Diet, Carbohydrate Restricted"OR “Diet, Low Carbohydrate"OR "Carbohydrate Diet, Low"OR "Carbohydrate Diets, Low" OR"Diets, Low Carbohydrate" OR "Low Carbohydrate Diets" OR "Carbohydrate-Restricted Diet" OR "Carbohydrate Restricted Diet" OR "Carbohydrate-Restricted Diets" OR "Diets, Carbohydrate-Restricted" OR"Low-Carbohydrate Diet" OR “Diet, Low-Carbohydrate" OR "Diets, Low-Carbohydrate" OR "Low Carbohydrate Diet"OR"Low-Carbohydrate Diets" OR “Diet, Gluten Free"OR "Gluten-Free Diet"OR“Diets, Gluten-Free"OR "Gluten Free Diet"OR "Gluten-Free Diets" OR "Ketogenic Diet"OR"Diets, Ketogenic" OR"Ketogenic Diets"OR"Diets, High-Fat" OR"High-Fat Diet" OR"High-Fat Diets" OR"Diet, High Fat" OR"Diets, High Fat"OR"High Fat Diet"OR"High Fat Diets"OR"Paleolithic Diet"OR "Diet, Stone Age"OR "Diets, Stone Age"OR "Stone Age Diet" OR "Stone Age Diets" OR "Diet, Paleo" OR"Diets, Paleo"OR "Paleo Diet"OR"Paleo Diets"OR"Diet, Caveman" OR "Caveman Diet"OR "Caveman Diets" OR"Diets, Caveman"OR"Diet, Hunter-Gatherer" OR"Diet, Hunter Gatherer" OR"Diets, Hunter-Gatherer"OR"Hunter-Gatherer Diet"OR"Hunter-Gatherer Diets"OR "Diets, Western"OR "Western Diets" OR"Western Dietary Pattern"OR "Dietary Pattern, Western"OR"Dietary Patterns, Western"OR"Pattern, Western Dietary" OR "Patterns, Western Dietary" OR "Western Dietary Patterns" OR"Diet, Occidental" OR"Diets, Occidental"OR "Occidental Diet" OR "Occidental Diets" OR"Western Diet" OR"Meat-Sweet Diet"OR"Diet, Meat-Sweet"OR "Diets, Meat-Sweet"OR"Meat Sweet Diet"OR"Meat-Sweet Diets") \#3 - (\#1 AND \#2) in trials

Lilacs (Por- (tw:("Lúpus Eritematoso Sistêmico" OU "Lúpus Eritematoso Sistêmico" OU “Lúpus Eritematoso Disseminado" OU "Doença de Libman-Sacks" OU tuguese) "Lupus Vulgaris" OU "Lúpus do Sistema Nervoso Central" OU "Lúpus Eritematoso Sistêmico do Sistema Nervoso Central" OU "Lúpus Eritematoso Sistêmico Neuropsiquiátrico" OU "Meningoencefalite Lúpica" OU “Meningoencefalites Lúpicas" OU "Paniculite Lúpica" OU "Paniculite Lúpicas" OU "Paniculite Lúpica Eritematosa" OU "Paniculite Lúpicas Eritematosas" OU "Lúpus Eritematoso Profundus" OU "Lupus Profundus" OU “Lúpus Eritematoso Discóide" OU “Lupus Eritematoso Crônico Cutâneo" OU "Lúpus Eritematoso Cutâneo Crônico" OU "Lúpus Eritematoso Cutâneo" OU "Lúpus Eritematoso Subcutâneo Cutâneo" OU "Lúpus Eritematoso Cutâneo Subagudo")) AND (tw:(Dieta OU Dietas)) AND (tw:( "Ensaios Clínicos Randomizados" OU "Ensaio Clínico Controlado Aleatório")) AND ( db:("LILACS"))

EMBASE (lupus OR 'lupus erythematosus'/exp OR 'lupus erythematosus' OR 'systemic lupus erythematosus'/exp OR 'systemic lupus erythematosus') AND ('nutrition'/exp OR nutrition OR 'diet, food, and nutrition' OR 'nutrition' OR 'nutrition council' OR 'nutrition phenomena' OR 'nutrition physiology' OR 'nutrition processes' OR 'nutrition research' OR 'nutrition research center' OR 'nutrition study' OR 'nutrition survey' OR 'nutrition surveys' OR 'nutritional physiological phenomena' OR 'nutritional physiology' OR 'nutritive solution' OR 'sports nutritional physiological phenomena' OR 'diet therapy'/exp OR 'diet intervention' OR 'diet therapy' OR 'diet treatment' OR 'dietary intervention' OR 'dietary therapy' OR 'dietary treatment' OR 'nutrition therapy' OR 'diet'/exp OR diet OR 'diet'OR 'diet influence' OR 'diet regimen' OR 'diet surveys' OR 'dietary effect' OR 'dietary influence' OR 'dietary survey' OR 'dietary surveys' OR 'dieting') AND ('controlled clinical trial'/exp OR 'clinical trial, controlled'OR 'controlled clinical comparison' OR 'controlled clinical drug trial' OR 'controlled clinical experiment' OR 'controlled clinical study' OR 'controlled clinical test' OR 'controlled clinical trial' OR 'randomized controlled trial'/exp OR 'controlled trial, randomized' OR 'randomised controlled study' OR 'randomised controlled trial' OR 'randomized controlled study' OR 'randomized controlled trial' OR 'trial, randomized controlled')

Open Grey "'("Lupus Erythematosus, Systemic"'"' OR "'"'Systemic Lupus Erythematogemsus"'"' OR "'"'Lupus Erythematosus Disseminatus"'") AND Search (Diet OR Diets OR "'"'nutritional management"'"' OR "'"'dietary manaent"'") AND "'("Clinical Trials, Randomized"'"' OR "'"'Trials, Randomized Clinical"'"' OR "'"'Controlled Clinical Trials, Randomized"'"' OR "'"'Randomized Controlled Trial

References 
outcomes. For binary data, a risk ratio (RR) with a 95\% confidence interval (CI) was used to assess the effect size. The risk difference between the intervention and control groups was calculated according to the Cochrane Collaboration handbook through the following calculus: $100 \times(1-\mathrm{RR}) \%$. Continuous data were evaluated through mean with a $95 \% \mathrm{CI}$. Due to the data heterogeneity, the meta-analysis was not possible to be performed; however, the effect size of the collected data was estimated with a 95\% CI and are presented in Table 2. Based on the Cochrane Collaboration handbook, for data interpretation, not only the statistical significance was considered, but also the change in the outcomes, which may not be relevant to the statistical aspect, but is relevant to the clinical aspect.

\section{Unit of analysis issues}

The unit of analysis was individual patients.

\section{Dealing with missing data}

In case of missing data, the authors of the studies would be consulted. However, it was not necessary, as the studies provided quantitative data.

\section{Studies summary}

The studies were summarized per author, publishing date, country, and place of study, intervention performed and results obtained.

\section{Risk of bias assessment and GRADE approach}

Through the Cochrane Collaboration risk-of-bias tool, the following domains were evaluated: random sequence generation, allocation sequence concealment, blinding of participants and personnel, blinding of reviewer, missing data, outcome selection, and other biases. Biases in texts included in the studies selection were identified. The risk of bias was evaluated by two independent reviewers (AMI and HES). There was no need for a third reviewer to resolve disagreements. The data was extracted from the selected articles, and the answers were classified using parameters of "yes", for low risk of bias; "no", for high risk of bias; and "unclear", for unknown or obscure risk of bias. The GRADE approach three domains and other considerations were used to grade the evidence for the main outcomes.

\section{Outcomes}

The potential significant outcomes were the evaluation of:
- Lipid profile parameters: High-density lipoprotein, Low-density lipoprotein, Triglyceride, Glycemic index)

- Disease activity, assessed with the Global activity by Systemic Lupus Erythematosus Disease Activity Index (SLEDAI), British Isles Lupus Assessment Group (BILAG), and European Community Lupus Activity Measure (ECLAM).

- Quality of life assessed with Lupus Quality of Life (LupusQoL), SLE-specific Quality of Life questionnaire (SLEQoL), SLE Quality of Life Questionnaire (L-QoL)), or another questionnaire with the same purpose.

- Quality and efficiency of sleep

- Fatigue with Fatigue Severity Scale (FSS).

The potential minor outcome was adherence to the diet.

\section{Results}

It was identified in the databases 758 articles; 132 were duplicated (Fig. 1); 616 references were screened, and 604 were excluded. After reading the title and abstract, 12 articles were included for full-text reading. After the fulltext reading, three studies were included for quantitative analysis. The reasons for the exclusion of nine articles from the study were: Alternative Treatments - antiseptics, antibiotics, supplementation $(\mathrm{n}=7)$; Cross-sectional, case-control, cohort studies, or any interventional design other than randomized clinical trials $(n=2)$.

The three articles included for the quantitative analysis were written in the United States $(\mathrm{n}=1)$, in Brazil $(\mathrm{n}=1)$, and in the United Kingdom $(\mathrm{n}=1)$ in 2002, 2012, and 2018, respectively. The sample size varied between 17 and 31 people diagnosed with systemic lupus erythematosus. The description of these studies is shown in Table 3.

\section{Sample characteristics}

Silva et al [11]. included adolescents diagnosed with systemic lupus erythematosus for at least six months, with any clinical manifestation and disease activity. Shah et al. [8] and Davies et al. [7] included only women diagnosed with systemic lupus erythematosus for at least six months. In the study by Davies et al. [7] there was no difference between the groups in the number of patients with hypertension and hyperlipidemia, nor was the number of caucasians and non-caucasians similar between the groups. Shah et al. [8] found that African American were $60 \%$ of both groups and the remainder, predominantly of Hispanic origin. 


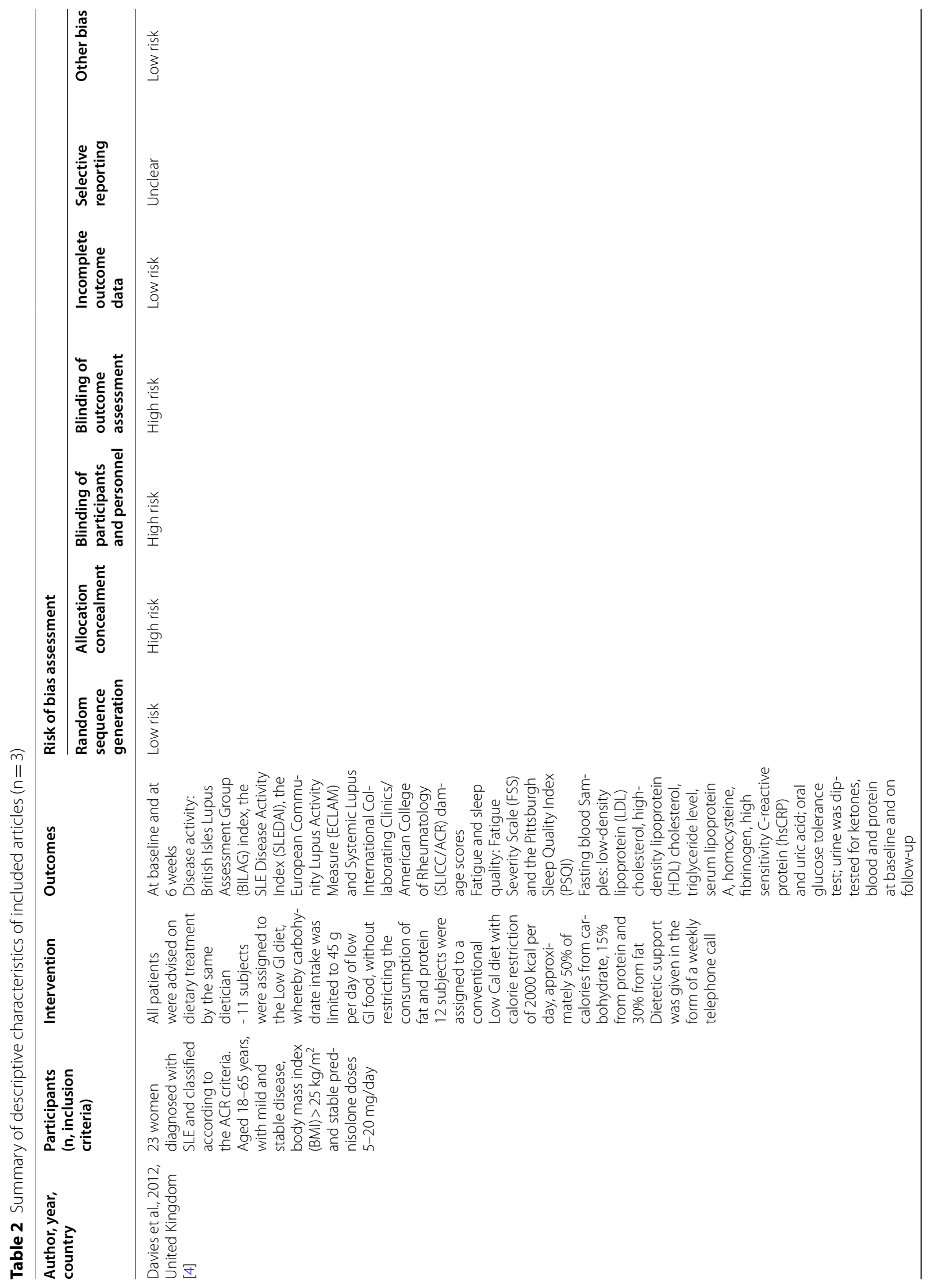




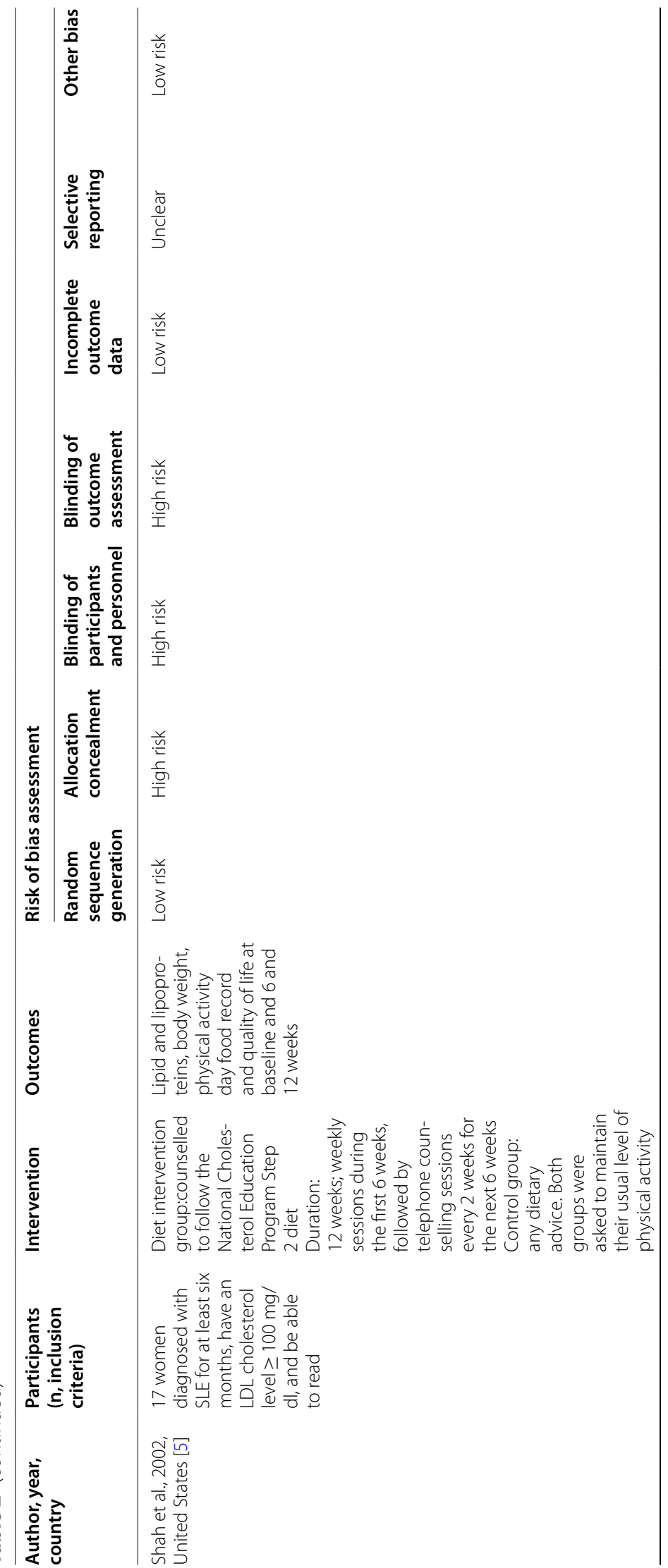




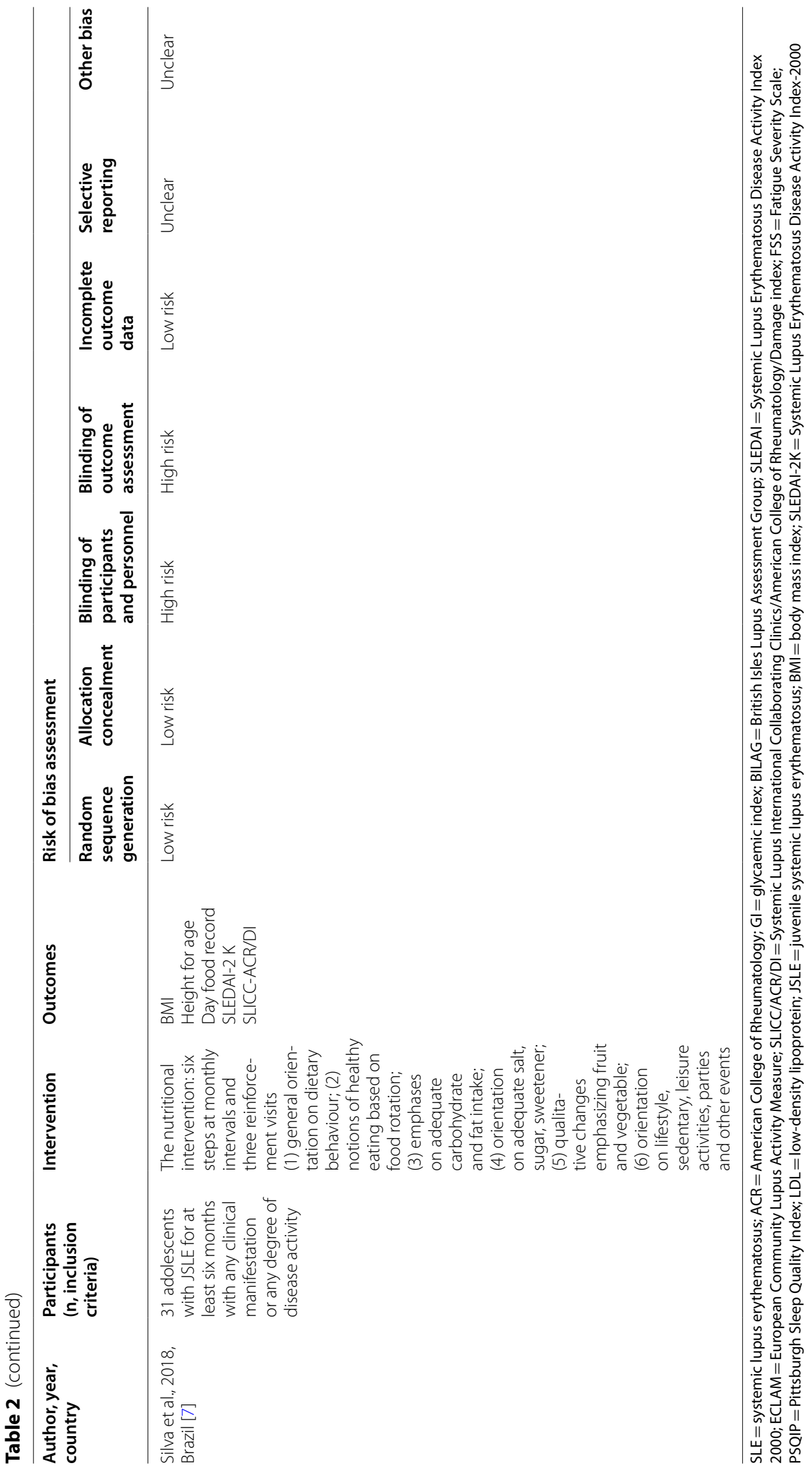




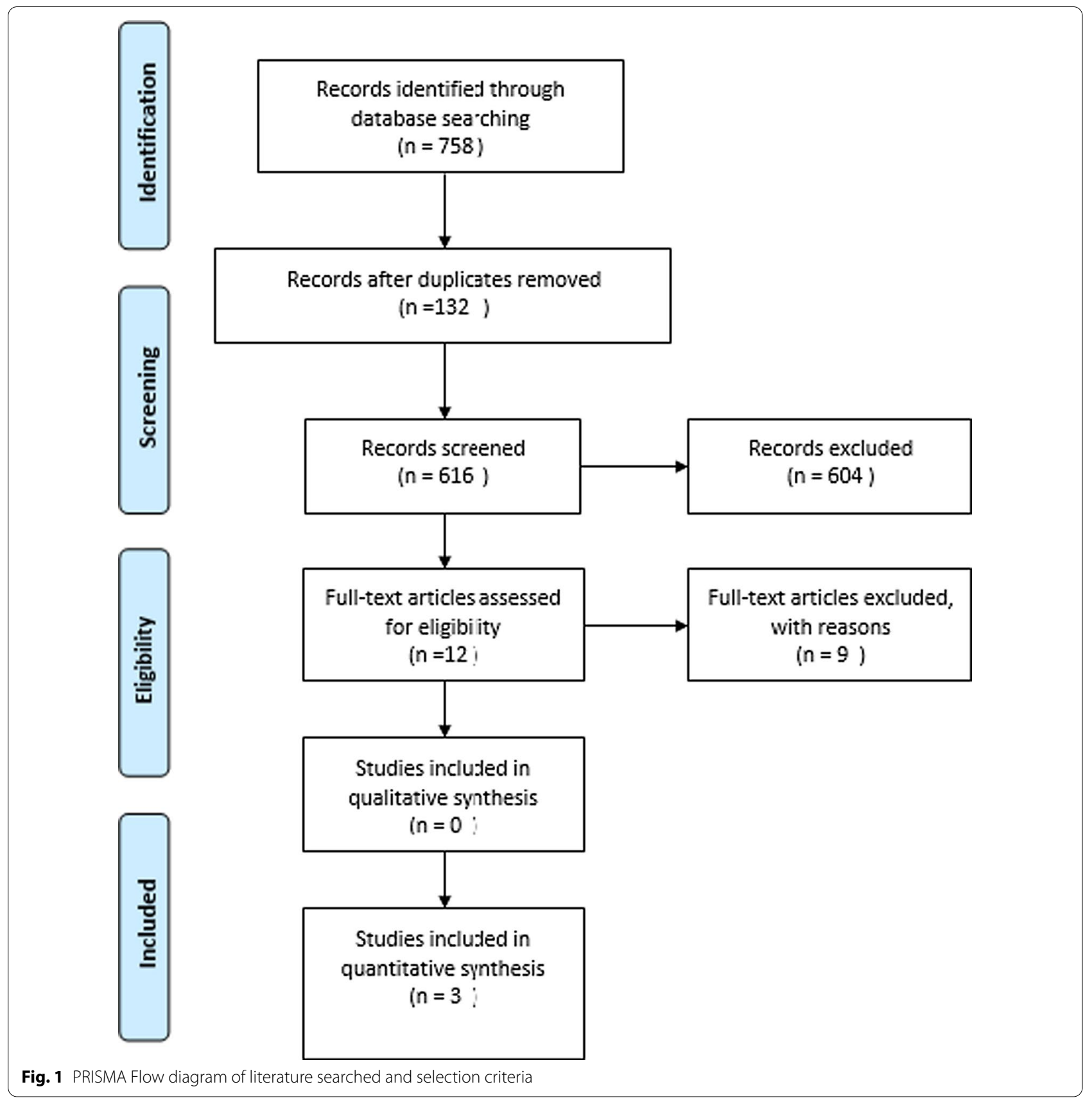

\section{Medication used by patients}

Regarding the use of medication, in the studies by Davies et al. [7] and Silva et al. [11], there was no significant difference between the groups in terms of prednisolone dosage and hydroxychloroquine users. Shah et al. [8] found that prednisolone use was slightly higher in the control group but without significant difference. In addition, four patients in the diet group and five patients in the control group were using cholesterol-lowering medication.

\section{Diet types}

The study conducted by Silva et al. [11] researched diet based on general behavioural guidelines, healthy eating, food diversity, balanced intake of fat and carbohydrate, and adequate intake of salt, fruit, vegetables, omega-3, and fibres. Additionally, orientations on lifestyle, frequency of meals, and physical activities were also analyzed. The controlled nutritional intervention study lasted nine months. 


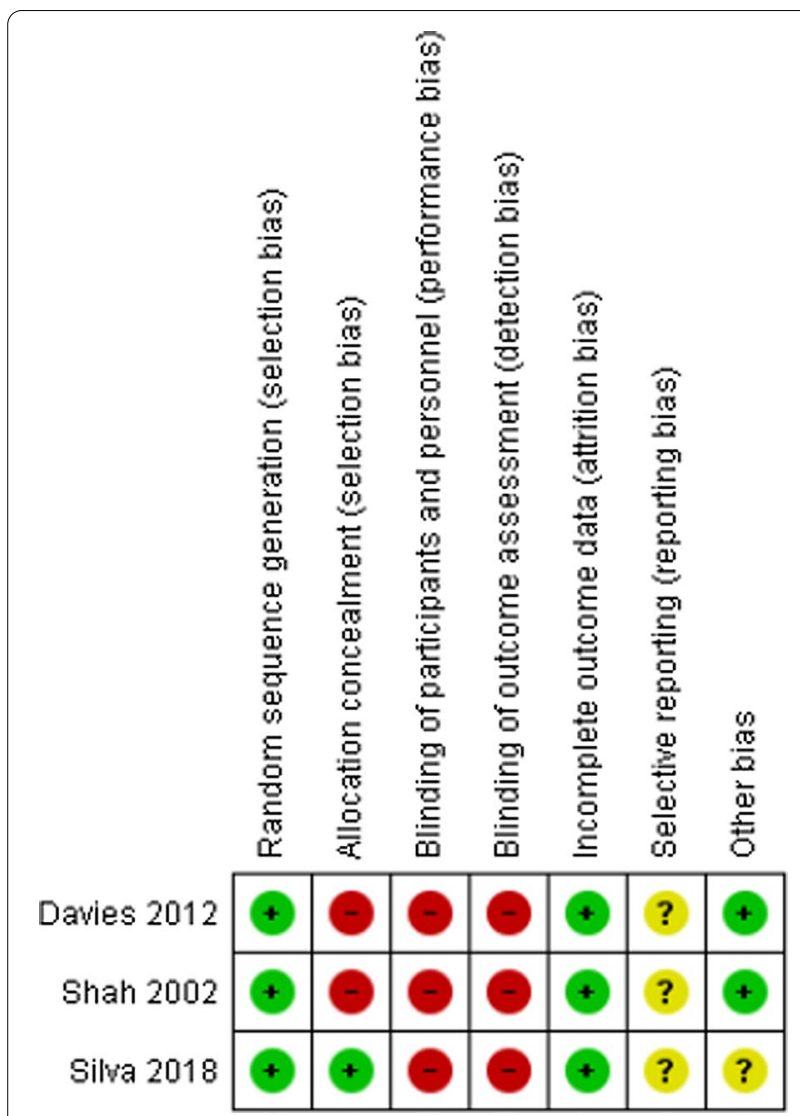

Fig. 2 Risk of bias assessment of included studies

Shah et al. [8] followed the National Cholesterol Education Program Step 2 diet: 30\% or less calories from fat ( $7 \%$ from saturated fat, $13 \%$ from monounsaturated fat, and $10 \%$ from polyunsaturated fat), and $<200 \mathrm{mg}$ of cholesterol per day. The diet intervention lasted for 12 weeks.

Davies et al. [7] compared two diet types. One diet type limited the carbohydrate intake to $45 \mathrm{~g}$ per day, without restricting the consumption of fat and protein. The composition of this diet was $10-15 \%$ of daily calories from carbohydrate, $25 \%$ from protein, and $60 \%$ from both saturated and unsaturated fat. The other was a calorie-restricted diet with limitation of $2000 \mathrm{kcal}$ per day, approximately $50 \%$ from carbohydrates, $15 \%$ from protein, and $30 \%$ from fat.

\section{Diet effects compared to control Quality of life}

Shah et al. [8] evaluated the quality of life using a selfadministered questionnaire. The diet produced a small contribution to the quality of life improvement at six weeks (mean difference (MD) 16.30; 95\% confidence interval (CI) 5.91; 26.69) and 12 weeks (MD 14.60; 95\%
CI $0.88 ; 28.32$ ). There was statistically significant difference in both times of evaluation.

\section{Disease activity}

Silva et al. [11] evaluated the disease activity through Systemic Lupus Erythematosus Disease Activity Index (SLEDAI) $2 \mathrm{~K}>4$. There was no relevant difference between both groups (RR 1.07; 95\% CI-0.32-3.52). There was no statistically significant difference.

\section{Lipid profile at six weeks}

Shah et al. [8] evaluated the lipid profile at six weeks. Although the diet caused a small improvement in the lipid profile of high-density lipoprotein (MD - 5.50; 95\% CI - 18.21; 7.21), low-density lipoprotein (MD 5.70; 95\% CI-16.72; 28.12), and triglyceride (MD - 2.50; 95\% CI - 70.67; 65.67), there was no statistically significant difference.

\section{Lipid profile at 12 weeks}

Shah et al. [8] evaluated the lipid profile at 12 weeks. The diet caused an improvement in the lipid profile of high-density lipoprotein (MD - 3.90; 95\% CI-17.54; 9.74), low-density lipoprotein (MD 15.30; 95\% $\mathrm{CI}-8.12$; 38.72), and triglyceride (MD - 13.40; $95 \%$ $\mathrm{CI}-50.27$; 23.47), however, there was no statistically significant difference.

\section{Lipid profile at nine months}

Silva et al. [11] evaluated the lipid profile at nine months. The low calorie diet was favorable for the improvement in high-density lipoprotein (MD 4.30; 95\% CI -6.96 ; $15.56 \mathrm{P}=0.45)$, low-density lipoprotein $(\mathrm{MD}-13.15 ; 95 \% \mathrm{CI}-29.38 ; 3.08 \quad \mathrm{P}=0.11)$, and triglyceride (MD 11.30; $95 \% \mathrm{CI}-16.90 ; 39.50$ $\mathrm{P}=0.43$ ), however, there was no statistically significant difference.

\section{Comparison between the low glycemic index diet and the calorie-restricted diet}

\section{Evaluation of disease activity, fatigue, and quality of sleep}

Davies et al. [7] evaluated the lipid profile at six weeks. The diet type did not affect the evaluation of fatigue, through the Fatigue Severity Scale (FSS) (MD 0.00; $95 \% \mathrm{CI}-1.20 ; 1.20 \mathrm{P}=0.00)$; disease activity, through the British Isles Lupus Assessment Group (BILAG) (MD 0.00; 95\% CI-3.52; $3.52 \mathrm{P}=1.00$ ); and quality of sleep, case on which it had small impact (MD 0.00; 95\% CI-3.52; $3.52 \mathrm{P}=1.00)$. Although there was no statistically significant difference, by evaluating the disease activity through the European Community 
Table 3 Post-intervention values of estimated effects for all outcomes evaluated

\begin{tabular}{|c|c|c|c|c|c|}
\hline Outcome & Studies & $\mathbf{N}$ & MD & Lower $95 \%$ & Higher $95 \%$ \\
\hline \multicolumn{6}{|l|}{ Diet effects compared to control } \\
\hline \multicolumn{6}{|l|}{ Quality of life } \\
\hline 6 weeks & 1 & 16 & 16.30 & 5.91 & 26.69 \\
\hline 12 weeks & 1 & 16 & 14.60 & 0.88 & 28.32 \\
\hline \multicolumn{6}{|l|}{ Lipid profile -6 weeks } \\
\hline $\mathrm{HDL}$ & 1 & 16 & 5.50 & -7.21 & 18.21 \\
\hline $\mathrm{LDL}$ & 1 & 16 & 5.70 & 16.72 & 28.12 \\
\hline Triglyceride & 1 & 16 & -2.50 & -70.67 & 65.67 \\
\hline \multicolumn{6}{|l|}{ Lipid profile - 12 weeks } \\
\hline $\mathrm{HDL}$ & 1 & 16 & 3.90 & 17.54 & 9.74 \\
\hline LDL & 1 & 16 & 15.30 & -8.12 & 38.72 \\
\hline Triglyceride & 1 & 16 & -13.40 & -50.27 & 23.47 \\
\hline \multicolumn{6}{|l|}{ Lipid profile - 9 months } \\
\hline $\mathrm{HDL}$ & 1 & 31 & 4.30 & -6.96 & 15.56 \\
\hline LDL & 1 & 31 & -13.15 & -29.38 & 3.08 \\
\hline \multirow[t]{2}{*}{ Triglyceride } & 1 & 31 & 11.30 & -16.90 & 39.50 \\
\hline & & & $\mathrm{RR}$ & Lower 95\% & Higher 95\% \\
\hline Disease activity (SLEDAI $2 \mathrm{~K}>4$ ) -6 weeks & 1 & 31 & 1.07 & 0.32 & 3.52 \\
\hline Outcome & Studies & $\mathbf{N}$ & MD & Lower $95 \%$ & Higher $95 \%$ \\
\hline \multicolumn{6}{|l|}{$\begin{array}{l}\text { Comparison between the low glycemic index diet and the } \\
\text { calorie-restricted diet }\end{array}$} \\
\hline \multicolumn{6}{|l|}{ Fatigue, quality of sleep, disease activity - 6 weeks } \\
\hline Fatigue Severity Scale & 1 & 23 & 0.00 & -1.20 & 1.20 \\
\hline Pittsburgh Sleep/Quality Index & 1 & 23 & 0.50 & -3.14 & 4.14 \\
\hline British Isles Lupus Assessment Group & 1 & 23 & 0.00 & -3.52 & 3.52 \\
\hline European Consensus Lupus Activity Measurement & 1 & 23 & -0.90 & -1.94 & 0.14 \\
\hline \multicolumn{6}{|l|}{ Lipid profile - 6 weeks } \\
\hline $\mathrm{HDL}$ & 1 & 23 & -0.24 & -0.48 & -0.00 \\
\hline LDL & 1 & 23 & -1.00 & -1.60 & -0.40 \\
\hline Triglyceride & 1 & 23 & -0.35 & -0.89 & 0.19 \\
\hline
\end{tabular}

$\mathrm{MD}=$ mean difference; $\mathrm{RR}=$ relative risk; $\mathrm{HDL}=$ high-density lipoprotein; $\mathrm{LDL}=$ low-density lipoprotein; $\mathrm{SLEDAI}=$ Systemic Lupus Erythematosus Disease Activity Index

Lupus Activity Measure (ECLAM), the low glycemic index diet showed a favourable effect (MD 0.90; 95\% $\mathrm{CI}-1.94 ; 0.14 \mathrm{P}=0.09)$.

\section{Lipid profile analyses}

Silva et al. [11] evaluated the lipid profile at six weeks. The low glycemic index diet was favorable for the improvement in high-density lipoprotein (MD - 0.24; 95\% CI $-0.48 ;-0.00 \mathrm{P}=0.05)$, low-density lipoprotein (MD - 1 ; 95\% CI $-1.60 ;-0.40 \mathrm{P}=0.001)$, and triglyceride ( $\mathrm{MD}-0.35 ; 95 \% \mathrm{CI}-0.89 ; 0.19 \mathrm{P}=0.20$ ). There was no statistically significant difference in the lowdensity lipoprotein evaluation $(\mathrm{P}=0.0001)$.

\section{Risk of bias and quality of evidence}

None of the studies met all methodological quality criteria. Two studies did not describe the concealment of the allocation sequence $[7,8]$, and none of the studies described the blinding of participants and personnel as determined by the Risk of Bias instrument used.

Three studies were considered to have a low risk of bias for incomplete outcomes and did not report the main predetermined result $[7,10]$. However, they were deemed to be free of other problems that could lead to a high risk of bias.

Quality of life, disease activity, and lipid profile outcomes at both 12 weeks and 9 months were selected and described as main outcomes in Table 4. Based on the risk of bias assessment and the GRADE approach three domains, it was found that through the evaluated 
outcomes, the level of evidence for the impact of diet on systemic lupus erythematosus patients is low (Fig. 2).

\section{Discussion}

The present study verified that the diet had a positive impact on the quality of life, and lipid profile of systemic lupus erythematosus patients. Three randomized controlled trials (RCTs) were included; however, due to the heterogeneity of the studies in relation to the control group and the moment of evaluation, it was not possible to perform the data meta-analysis. Nonetheless, to verify the effect size of interventions and compare it among the groups, the data presentation of choice was by effect size estimation.

According to Shah et al. [8], the diet intervention presented a small effect on the quality of life improvement. The questionnaire used by the authors assessed the general energy level, ability to perform specific tasks, quality of sleep, satisfaction with food eaten, satisfaction with personal life, relationships, etc. According to Chaigne et al. [12], musculoskeletal involvement, sociodemographic factors, fatigue, and comorbidities are directly related to the quality of life of systemic lupus erythematosus patients and can be modified when subjected to a controlled diet.

The European League Against Rheumatism (EULAR) stated that the quality of life-related to health is an important parameter and should be continuously evaluated in the clinical routine. Regarding the quality of life, the authors did not attribute a slight improvement to the diet itself, but the intensity of counselling received by patients in the intervention group.

The disease activity assessed by Systemic Lupus Erythematosus Disease Activity Index $2 \mathrm{~K}>4$ at the end of the study conducted by Silva et al. [11], which lasted for six months, did not show any relevant difference between the group submitted to the diet and the control group. According to the authors, the groups did not differ regarding the corticoid dose used throughout the study. The authors observed that there was a significant change in the intervention group, with a reduction in the carbohydrate, saturated fat, trans and total fat, and calories intake. There was no change in the body mass index among participants in the intervention group, but when compared to the control group, these participants presented a weight gain. As the adipose tissue secretes pro-inflammatory cytokines, the increased adipose mass may contribute to the inflammation worsening, which may justify the disease activity worsening in the control group [13].

The subject is still controversial, but there is a suggestion that obesity and a high-fat diet are related to
SLE, with the frequency of obesity being higher in SLE patients than in the general population. Vitamin D deficiency and increased adipokines, such as leptin and adiponectin, may explain the relationship between obesity and SLE [14].

Regarding the lipid profile, the study conducted by Shah et al. [8] showed that the diet improved the highdensity lipoprotein, low-density lipoprotein, and triglyceride levels at both 6 and 12 weeks, which was more accentuated at 12 weeks. Silva et al. [11] showed an improvement in high-density lipoprotein, low-density lipoprotein, and triglyceride levels in adolescents at the 9-month evaluation. The results of both studies prove the effectiveness of protecting the cardiovascular system through diet and that adults and adolescents adhered to the proposed diet. For involving adolescents, the study conducted by Silva et al. [11] proves that the diet can be effective even among groups that are usually resistant to changing habits [15]. According to the Update of the Brazilian Guideline on Dyslipidemias and Prevention of Atherosclerosis, the control of saturated fat intake and its substitution with polyunsaturated fatty acids, precepts followed in both studies, are associated with cholesterol total and low-density lipoprotein cholesterol decrease, as well as with a decrease in cardiovascular events and death [16].

The comparison between the diets was also enabled by Davies et al. study [7], which compared the low glycemic index and calorie-restricted diets. As for the lipid profile, Davies et al. [7] verified that none of the groups presented a significant index reduction when compared to the initial evaluation. However, when the data was inserted in the forest plot, it shows that the low glycemic index diet was more favourable for high-density lipoprotein, low-density lipoprotein, and triglyceride improvement than the calorie-restricted diet. The type of diet did not affect the fatigue, quality of sleep, and disease activity as assessed through the British Isles Lupus Assessment Group. However, when the European Community Lupus Activity Measure evaluated the low glycemic index diet, its effect was favourable on fatigue. The study conducted by Davies et al. [7] shows that the low glycemic index diet redresses the postprandial hyperglycemia and hyperinsulinemia and the late postprandial hypoglycemia, which increases the risk of obesity, diabetes, and cardiovascular disease. The Update of the Brazilian Guideline on Dyslipidemias and Prevention of Atherosclerosis indicates as an adequate strategy for handling dyslipidemia diets such as the Mediterranean diet, emphasizing the importance of keeping a moderate-fat quantity, eliminating trans fatty acids, controlling the consumption of saturated fats, prioritizing polyunsaturated and monounsaturated 
Table 4 Summary of findings

Question: What is the diet effect on systemic lupus erythematosus (SLE) patients?

Patients or population: Patients diagnosed with SLE

Setting: Ambulatory care

Intervention: Diet

Comparison: No diet

\begin{tabular}{|c|c|c|c|c|c|c|}
\hline Outcomes & $\begin{array}{l}\text { Illustrative com } \\
\mathbf{9 6 \%} \% \mathbf{C I}) \\
\text { Assumed risk } \\
\text { Corresponding r } \\
\text { Control } \\
\text { Diet } \\
\end{array}$ & $\begin{array}{l}\text { parative risk } \\
\text { sk }\end{array}$ & Relative effect & $\begin{array}{l}\text { No of } \\
\text { participants } \\
\text { (studies) }\end{array}$ & $\begin{array}{l}\text { Quality of } \\
\text { Evidence } \\
\text { (GRADE) }\end{array}$ & Comments \\
\hline $\begin{array}{l}\text { Quality of life: } 12 \text { weeks } \\
\text { Range: 0-100 } \\
\text { An increased score } \\
\text { reflects improved QOL }\end{array}$ & $\begin{array}{l}\text { The mean } \\
\text { quality of life } \\
\text { in the control } \\
\text { groups was } \\
53.8 \text {. }\end{array}$ & $\begin{array}{l}\text { The mean } \\
\text { quality of life in } \\
\text { the intervention } \\
\text { groups was MD } \\
14.6 \text { higher } \\
\text { (0.88 higher to } \\
28.32 \text { higher) }\end{array}$ & & $\begin{array}{l}16 \\
\text { (1 study) }\end{array}$ & $\begin{array}{l}\oplus \oplus \underset{\infty}{\mathrm{LOW}^{2, b}} \\
\end{array}$ & $\begin{array}{l}\text { Risk of bias: serious } \\
\text { Imprecision: serious } \\
\text { The author of the study named didn't specify the } \\
\text { tool used to evaluate the quality of life }\end{array}$ \\
\hline $\begin{array}{c}\text { Disease activity } \\
\text { (SLEDAI 2K>4): } \\
\text { six weeks }\end{array}$ & $\begin{array}{l}266 \text { per } \\
1000\end{array}$ & $\begin{array}{l}\text { 278 per 1000 } \\
\text { (83 to } 915)\end{array}$ & $\begin{array}{l}\text { RR } 1.07 \\
\text { (0.32 to } 3.52)\end{array}$ & $\begin{array}{l}31 \\
\text { (1 study) }\end{array}$ & $\begin{array}{l}\oplus \oplus \mathscr{\infty} \\
\text { LOW }^{, d}\end{array}$ & $\begin{array}{l}\text { Risk of bias: serious } \\
\text { Imprecision: serious }\end{array}$ \\
\hline $\begin{array}{l}\text { Range: } \\
0-105 . \\
\text { higher value higher } \\
\text { disease activity. } \\
\text { a value above } 6 \\
\text { indicates a significant } \\
\text { clinical change } \\
\text { sufficient to change the } \\
\text { management }\end{array}$ & & & & & & \\
\hline $\begin{array}{l}\text { HDL: } 12 \text { weeks } \\
\text { Higher is better }\end{array}$ & $\begin{array}{l}\text { The mean } \\
\text { HDL leve in } \\
\text { the control } \\
\text { groups was } \\
49.4\end{array}$ & $\begin{array}{l}\text { The mean HDL } \\
\text { level in the } \\
\text { intervention } \\
\text { groups was MD } \\
3.9 \text { higher } \\
9.74 \text { lower to } \\
17.54 \text { higher) }\end{array}$ & & $\begin{array}{l}16 \\
\text { (1 study) }\end{array}$ & 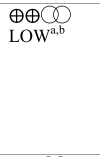 & $\begin{array}{l}\text { Risk of bias: serious } \\
\text { Imprecision: serious }\end{array}$ \\
\hline $\begin{array}{l}\text { Total cholesterol: } 12 \\
\text { weeks } \\
\text { Higher is worse }\end{array}$ & $\begin{array}{l}\text { The mean total } \\
\text { cholesterol in } \\
\text { the control } \\
\text { groups was } \\
194.3 \text {. }\end{array}$ & $\begin{array}{l}\text { The mean total } \\
\text { cholesterol } \\
\text { level in the } \\
\text { intervention } \\
\text { groups was MD } \\
15.8 \text { higher } \\
(8.46 \text { lower to } \\
40.06 \text { higher) }\end{array}$ & ---o- & $\begin{array}{l}16 \\
\text { (1 study) }\end{array}$ & $\begin{array}{l}\oplus \oplus \underset{⿴ 囗 十}{L^{\mathrm{a}, \mathrm{e}}} \\
\end{array}$ & $\begin{array}{l}\text { Risk of bias: serious } \\
\text { Imprecision: serious }\end{array}$ \\
\hline $\begin{array}{l}\text { Triglyceride: } 12 \text { weeks } \\
\text { Higher is worse }\end{array}$ & $\begin{array}{l}\text { The mean } \\
\text { triglyceride } \\
\text { level in the } \\
\text { control groups } \\
\text { was } 128 .\end{array}$ & $\begin{array}{l}\text { The mean } \\
\text { triglyceride } \\
\text { level in the } \\
\text { intervention } \\
\text { groups was MD } \\
\text { 13.4 lower } \\
\text { (50.27 lower to } \\
\text { 23.47 higher) }\end{array}$ & & $\begin{array}{l}16 \\
\text { (1 study) }\end{array}$ & $\begin{array}{l}\oplus \oplus \infty \\
\text { LOW }^{2, e}\end{array}$ & $\begin{array}{l}\text { Risk of bias: serious } \\
\text { Imprecision: serious }\end{array}$ \\
\hline $\begin{array}{l}\text { LDL: } 12 \text { weeks } \\
\text { Higher is worse }\end{array}$ & $\begin{array}{l}\text { The mean LDL } \\
\text { level in the } \\
\text { control groups } \\
\text { was } 128 \text {. }\end{array}$ & $\begin{array}{l}\text { The mean LDL } \\
\text { level in the } \\
\text { intervention } \\
\text { groups was MD } \\
15.3 \text { higher } \\
(8.12 \text { lower to } \\
38.72 \text { higher) }\end{array}$ & - & $\begin{array}{l}16 \\
\text { (1 study) }\end{array}$ & $\begin{array}{l}\oplus \oplus{ }^{\oplus} \\
\text { LOW }^{\text {a,e }}\end{array}$ & $\begin{array}{l}\text { Risk of bias: serious } \\
\text { Imprecision: serious }\end{array}$ \\
\hline $\begin{array}{l}\text { Total cholesterol: nine } \\
\text { months } \\
\text { Higher is worse }\end{array}$ & $\begin{array}{l}\text { The mean total } \\
\text { cholesterol } \\
\text { level in the } \\
\text { control groups } \\
\text { was } 160.2\end{array}$ & $\begin{array}{l}\text { The mean total } \\
\text { cholesterol at } \\
\text { nine months in } \\
\text { the intervention } \\
\text { groups was MD } \\
\text { 15.3 lower } \\
\text { (36.35 lower to } \\
5.75 \text { higher) }\end{array}$ & & $\begin{array}{l}31 \\
\text { (1 study) }\end{array}$ & $\begin{array}{l}\oplus \oplus \infty \\
\text { LOW }^{a, b}\end{array}$ & $\begin{array}{l}\text { Risk of bias: serious } \\
\text { Imprecision: serious }\end{array}$ \\
\hline $\begin{array}{l}\text { LDL: nine months } \\
\text { Higher is worse }\end{array}$ & $\begin{array}{l}\text { The mean LDI } \\
\text { level in the } \\
\text { control groups } \\
\text { was } 87.75\end{array}$ & $\begin{array}{l}\text { The mean LDL } \\
\text { level at nine } \\
\text { months in the } \\
\text { intervention } \\
\text { groups was MD } \\
13.15 \text { lower } \\
\text { (29.38 lower to } \\
3.08 \text { higher) }\end{array}$ & & $\begin{array}{l}31 \\
(1 \text { study })\end{array}$ & 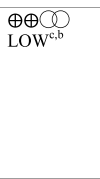 & $\begin{array}{l}\text { Risk of bias: serious } \\
\text { Imprecision: serious }\end{array}$ \\
\hline $\begin{array}{l}\text { Triglyceride: nine } \\
\text { months } \\
\text { Higher is worse }\end{array}$ & $\begin{array}{l}\text { The mean } \\
\text { triglyceride } \\
\text { level in the } \\
\text { control groups } \\
\text { was } 87.2\end{array}$ & $\begin{array}{l}\text { The mean } \\
\text { triglyceride } \\
\text { level at nine } \\
\text { month in the } \\
\text { intervention } \\
\text { groups was MD } \\
11.3 \text { higher } \\
\text { (16.9 lower to } \\
\text { 39.5 higher) }\end{array}$ & & $\begin{array}{l}31 \\
\text { (1 study) }\end{array}$ & $\begin{array}{l}\oplus \oplus \mathscr{C}^{\mathrm{L}} \\
\mathrm{LOW}^{\mathrm{b}, \mathrm{b}}\end{array}$ & $\begin{array}{l}\text { Risk of bias: serious } \\
\text { Imprecision: serious }\end{array}$ \\
\hline $\begin{array}{l}\text { HDL: nine months } \\
\text { Higher is worse }\end{array}$ & $\begin{array}{l}\text { The mean } \\
\text { HDL level in } \\
\text { the control } \\
\text { groups was } 54\end{array}$ & $\begin{array}{l}\text { The mean HDL } \\
\text { level at nine } \\
\text { months in the } \\
\text { intervention } \\
\text { groups was MD } \\
4.3 \text { lower } \\
\text { (15.56 lower to } \\
6.96 \text { higher) }\end{array}$ & & $\begin{array}{l}31 \\
(1 \text { study })\end{array}$ & $\begin{array}{l}\oplus \oplus \mathscr{D} \\
\mathrm{LOW}^{\mathrm{b}, b}\end{array}$ & $\begin{array}{l}\text { Risk of bias: serious } \\
\text { Imprecision: serious }\end{array}$ \\
\hline
\end{tabular}


Table 4 (continued)

The basis for the assumed risk (e.g. the median control group risk across studies) is provided in footnotes. The corresponding risk (and its $95 \%$ confidence interval) is based on the assumed risk in the comparison group and the relative effect of the intervention (and its $95 \% \mathrm{Cl}$ )

$\mathrm{Cl}=$ confidence interval; $\mathrm{MD}=$ mean difference; $\mathrm{RR}=$ risk ratio; $\mathrm{SLEDAI}=$ Systemic Lupus Erythematosus Disease Activity Index; $\mathrm{HDL}=$ high-density lipoprotein; $\mathrm{LDL}=$ low-density lipoprotein; $\mathrm{a}=$ There is a selection and detection bias; $b=$ Small sample size; $c=$ Detection bias; $d=$ Small sample size and small number of events; $\mathrm{e}=$ Few patients and very large confidence interval $(>0.5)$

fats, reducing sugars, and including lean meats, fruits, grains, and vegetables in the diet [16]. It further stresses that high carbohydrate intake increases glycemia, which generates hyperinsulinemia. In turn, this condition activates the transcription factors that promote the synthesis of fatty acids and triglycerides. There is still no substantial evidence proving the effectiveness of the low carbohydrate diet on lipid profile, mainly because the studies diverge significantly on the low carbohydrate diet definition. The 2018 United Kingdom guidelines on diabetes show that long-term adherence to low carbohydrate diets is low [17].

It has been demonstrated in studies that medications such as hydroxychloroquine and prednisone may affect the lipid profile $[18,19]$. Hydroxychloroquine seems to have a beneficial effect due to the reduction of atherogenic lipoproteins and prednisone is associated with a worsening in the lipid profile of patients with lupus activity [18]. The studies included in this review did not differ between groups regarding the use of hydroxychloroquine and prednisone. It is important to note that the review evaluated patients with inactive disease and, probably, with low doses of these drugs.

Medeiros et al. [] published a systematic review investigating the effects of nutritional supplements such as omega- 3 and vitamin $\mathrm{D}$, as well as the study by Islam et al. who recommends intake of Omega 3 and 6; vitamins A, $\mathrm{B}, \mathrm{C}, \mathrm{D}$, and $\mathrm{E}$; minerals (calcium, zinc, selenium, iron, and copper) and polyphenol-containing foods to produce an immunomodulatory effect in SLE patients [21], differing from the present study because the objective of the latter was to evaluate the nutritional diet impact, not a specific nutritional supplement. Regarding methodological aspects, the review by Medeiros et al. [] restricted the search to the period from 2006 to 2016, and the exclusion criteria evaluated nutrients or diet analyses only. Furthermore, the systematic review did not describe the results regarding the effectiveness of the interventions.

Supplementation of macronutrients and micronutrients produces immunomodulatory effects in SLE patients, so the intake of Omega 3 and 6, vitamins A, B, $\mathrm{C}, \mathrm{D}$, and $\mathrm{E}$, minerals such as calcium, zinc, selenium, iron, and copper, and polyphenol-containing foods are recommended.

Because dietary supplementation of various macro and micronutrients have exhibited immunomodulatory effects, including maintenance of homeostasis and improved physical and mental well-being in SLE patients, it is recommended that these patients consume a balanced diet that is low in calories and protein but contains plenty of fiber, PUFAs ( $\omega 3$ and $\omega-6)$, vitamins (A, B, C, D, and $\mathrm{E}$ ), minerals (calcium, zinc, selenium, iron, and copper), and polyphenol-containing foods.

The present systematic review is the first study about the nutritional diet impact on systemic lupus erythematosus patients and followed the methodological principles considered essential to conduct and report a systematic review, which includes conducting searches in several databases without date and language restriction, evaluating and selecting articles independently, and registering the protocol on PROSPERO.

Some limitations were found in this review. The small number of included studies and the small number of patients included can lead to a conclusion that does not correspond to reality. The different types of diets evaluated makes it difficult to standardize the studied intervention. This problem can be overcome by conducting studies with the involvement of several centres in a multicenter way. Also, the heterogeneity of the data makes it impossible to carry out a meta-analysis. Due to these limitations, further studies are needed to confirm the association between the impact of diet on patients with systemic lupus erythematosus.

\section{Conclusions}

Regarding its implication for the practice, studies such as the present systematic review help to promote public policies focusing on the population with rare and chronic diseases such as systemic lupus erythematosus. As for its implication for research, being the proposed interventions safe and effective, it should encourage researches aimed at verifying the outcome of the specific nutritional diet implementation in systemic lupus erythematosus patients.

The diet had a positive impact on the quality of life and a possible clinical relevance on lipid profile (high-density lipoprotein, low-density lipoprotein, triglyceride levels) of systemic lupus erythematosus patients. Both types of diet, calorie-restricted and low glycemic index did not influence disease activity, fatigue and quality of sleep 
outcomes. Based on GRADE approach, the evidence level for all outcomes was considered low.

\section{Acknowledgements}

Not applicable.

\section{Authors' contributions}

Conceptua Imoto AM lization (lead), data curation (lead), formal analysis (lead), funding acquisition (lead), methodology (lead), project administration (lead), supervision (lead), validation (lead), visualization (lead), writing-original draft (lead), writing-review and editing (lead); Gottems L: funding acquisition (supporting), writing-original draft (supporting), writing-review and editing (supporting); Salomon AL: conceptualization (supporting), data curation (supporting), writing-original draft (supporting), writing-review and editing (supporting); Silva HEC: conceptualization (supporting), data curation (supporting), funding acquisition (supporting), methodology (supporting), writing-original draft (supporting); Lombardi Júnior l: writing-review and editing (supporting); Peccin MS: writing-review and editing (supporting); Amorim FF: writingoriginal draft (supporting), writing-review and editing (supporting); Santana LA: writing-review and editing (supporting). All authors read and approved the final manuscript.

\section{Funding}

Fundação de Ensino e Pesquisa em Ciências da Saúde (00064-0000417/2018-52).

\section{Availability of data and materials}

Not applicable.

\section{Declarations}

\section{Ethics approval and consent to participate}

Not applicable.

\section{Consent for publication}

Not applicable.

\section{Competing interests}

All authors declare that they have no competing interests.

\section{Author details}

${ }^{1}$ Evidences-Based Health Lab, Professional and Academic Master Program, Escola Superior em Ciências da Saúde, Brasília, DF, Brazil. ${ }^{2}$ Professional and Academic Master Program, Escola Superior em Ciências da Saúde, Brasília, DF, Brazil. ${ }^{3}$ Human Movement Sciences Department, Interdisciplinary Post-Graduation Program in Health Sciences, Universidade Federal de São Paulo (UNIFESP), Santos, SP, Brazil. ${ }^{4}$ Medicine Graduation Course, Escola Superior em Ciências da Saúde, Brasília, DF, Brazil. ${ }^{5}$ Family Health Master Program (ProfSaúde), Escola Superior em Ciências da Saúde, Brasília, DF, Brazil.

Received: 14 May 2021 Accepted: 17 October 2021

Published online: 06 November 2021

\section{References}

1. Zucchi D, Elefante E, Calabresi E, et al. One year in review 2019: systemic lupus erythematosus. Clin Exp Rheumatol. 2019;37(5):715-22.

2. Lupus Commission. Systemic lupus erythematosus (SLE): BSR primer. Brazilian Society of Rheumatology. 2011. http://reumatologia.org.br/ orientacoes-ao-paciente/lupus-eritematoso-sistemico-les-cartilha-da-sbr. Accessed 08 Oct 2020.

3. Rees F, Doherty M, Grainge MJ, Lanyon P, Zhang W. The worldwide incidence and prevalence of systemic lupus erythematosus: a systematic review of epidemiological studies. Rheumatology (Oxford). 2017;56(11):1945-61. https://doi.org/10.1093/rheumatology/kex260.

4. Kiriakidou M, Ching CL. Systemic lupus erythematosus. Ann Intern Med. 2020;172(11):ITC81-96. https://doi.org/10.7326/AITC202006020.
5. Petri M, Orbai AM, Alarcón GS, et al. Derivation and validation of the Systemic Lupus International Collaborating Clinics classification criteria for systemic lupus erythematosus. Arthritis Rheum. 2012;64(8):2677-86. https://doi.org/10.1002/art.34473.

6. Klack K, Bonfa E, Borba Neto EF. Diet and nutritional aspects in systemic lupus erythematosus. Rev Bras Reumatol. 2012;52(3):384-408. https://doi. org/10.1590/S0482-50042012000300009.

7. Davies RJ, Lomer MC, Yeo SI, et al. Weight loss and improvements in fatigue in systemic lupus erythematosus: a controlled trial of a low glycaemic index diet versus a calorie restricted diet in patients treated with corticosteroids. Lupus. 2012;21(6):649-55. https://doi.org/10.1177/09612 03312436854.

8. Shah M, Kavanaugh A, Coyle Y, Adams-Huet B, Lipsky PE. Effect of a culturally sensitive cholesterol lowering diet program on lipid and lipoproteins, body weight, nutrient intakes, and quality of life in patients with systemic lupus erythematosus. J Rheumatol. 2002;29(10):2122-8.

9. Aparicio-Soto M, Sánchez-Hidalgo M, Alarcón-de-la-Lastra C. An update on diet and nutritional factors in systemic lupus erythematosus management. Nutr Res Rev. 2017;30(1):118-37. https://doi.org/10.1017/S0954 422417000026.

10. La Cava A. The influence of diet and obesity on gene expression in SLE. Genes (Basel). 2019;10(5):405. https://doi.org/10.3390/genes10050405.

11. da Silva SGL, Terreri MT, Abad TTO, et al. The effect of nutritional intervention on the lipid profile and dietary intake of adolescents with juvenile systemic lupus erythematosus: a randomized, controlled trial. Lupus. 2018;27(5):820-7. https://doi.org/10.1177/0961203317751851.

12. Chaigne $B$, Chizzolini $C$, Perneger $T$, et al. Impact of disease activity on health-related quality of life in systemic lupus erythematosus - a crosssectional analysis of the Swiss Systemic Lupus Erythematosus Cohort Study (SSCS). BMC Immunol. 2017;18(1):17. https://doi.org/10.1186/ s12865-017-0200-5.

13. Coppack SW. Pro-inflammatory cytokines and adipose tissue. Proc Nutr Soc. 2001;60(3):349-56. https://doi.org/10.1079/pns2001110.

14. Kono $M$, Nagafuchi $Y$, Shoda $H$, et al. The impact of obesity and a high-fat diet on clinical and immunological features in systemic lupus erythematosus. Nutrients. 2021;13(2):504. https://doi.org/10.3390/nu13020504.

15. Ho M, Garnett SP, Baur L, et al. Effectiveness of lifestyle interventions in child obesity: systematic review with meta-analysis. Pediatrics. 2012;130(6):e1647-71. https://doi.org/10.1542/peds.2012-1176.

16. Faludi AA, Izar MCO, Saraiva JFK, et al. Atualização da Diretriz Brasileira de Dislipidemias e Prevenção da Aterosclerose - 2017. Arq Bras Cardiol. 2017;109(2 Supl 1):1-76. https://doi.org/10.5935/abc.20170121.

17. Dyson PA, Twenefour D, Breen C, et al. Diabetes UK evidence-based nutrition guidelines for the prevention and management of diabetes. Diabet Med. 2018;35(5):541-7. https://doi.org/10.1111/dme.13603.

18. Cairoli E, Rebella M, Danese N, Garra V, Borba EF. Hydroxychloroquine reduces low-density lipoprotein cholesterol levels in systemic lupus erythematosus: a longitudinal evaluation of the lipid-lowering effect. Lupus. 2012;21(11):1178-82. https://doi.org/10.1177/0961203312450084.

19. Sarkissian T, Beyene J, Feldman B, McCrindle B, Silverman ED. Longitudinal examination of lipid profiles in pediatric systemic lupus erythematosus. Arthritis Rheum. 2007;56(2):631-8. https://doi.org/10.1002/art.22332.

20. Durcan L, Winegar DA, Connelly MA, et al. Longitudinal evaluation of lipoprotein variables in systemic lupus erythematosus reveals adverse changes with disease activity and prednisone and more favorable profiles with hydroxychloroquine therapy. J Rheumatol. 2016;43(4):745-50. https://doi.org/10.3899/jrheum.150437.

21. Islam MA, Khandker SS, Kotyla PJ, Hassan R. Immunomodulatory effects of diet and nutrients in systemic lupus erythematosus (SLE): a systematic review. Front Immunol. 2020;11:1477. https://doi.org/10.3389/fimmu. 2020.01477.

\section{Publisher's Note}

Springer Nature remains neutral with regard to jurisdictional claims in published maps and institutional affiliations. 